\title{
Single Residue on the WPD-Loop Affects the pH Dependency of Catalysis in Protein Tyrosine Phosphatases
}

\author{
Ruidan Shen, Rory M. Crean, Sean J. Johnson,* Shina C. L. Kamerlin,* and Alvan C. Hengge* \\ Cite This: JACS Au 2021, 1, 646-659 \\ Read Online
}

ABSTRACT: Catalysis by protein tyrosine phosphatases (PTPs) relies on the motion of a flexible protein loop (the WPD-loop) that carries a residue acting as a general acid/base catalyst during the PTP-catalyzed reaction. The orthogonal substitutions of a noncatalytic residue in the WPD-loops of YopH and PTP1B result in shifted $\mathrm{pH}$-rate profiles from an altered kinetic $\mathrm{p} K_{\mathrm{a}}$ of the nucleophilic cysteine. Compared to wild type, the G352T YopH variant has a broadened $\mathrm{pH}$-rate profile, similar activity at optimal $\mathrm{pH}$, but significantly higher activity at low $\mathrm{pH}$. Changes in the corresponding PTP1B T177G variant are more modest and in the opposite direction, with a narrowed $\mathrm{pH}$ profile and less activity in

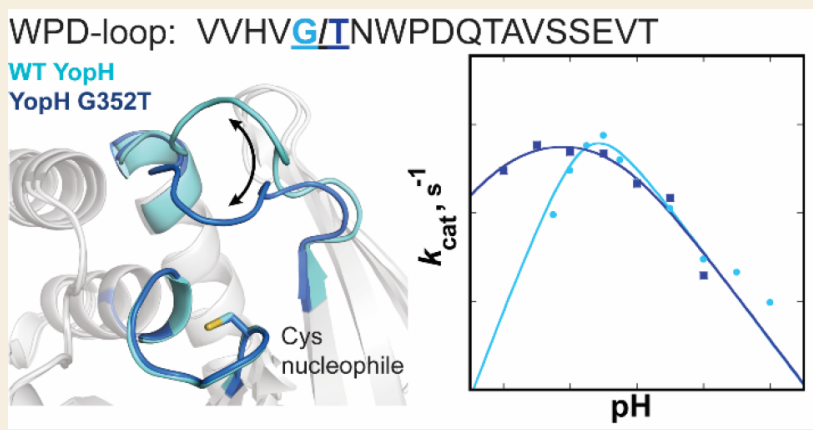
the most acidic range. Crystal structures of the variants show no structural perturbations but suggest an increased preference for the WPD-loop-closed conformation. Computational analysis confirms a shift in loop conformational equilibrium in favor of the closed conformation, arising from a combination of increased stability of the closed state and destabilization of the loop-open state. Simulations identify the origins of this population shift, revealing differences in the flexibility of the WPD-loop and neighboring regions. Our results demonstrate that changes to the $\mathrm{pH}$ dependency of catalysis by PTPs can result from small changes in amino acid composition in their WPD-loops affecting only loop dynamics and conformational equilibrium. The perturbation of kinetic $\mathrm{p} K_{\mathrm{a}}$ values of catalytic residues by nonchemical processes affords a means for nature to alter an enzyme's $\mathrm{pH}$ dependency by a less disruptive path than altering electrostatic networks around catalytic residues themselves.

KEYWORDS: Protein tyrosine phosphatases, Enzyme kinetics, Protein dynamics, Enzyme catalysis, Point mutation, Loop dynamics, $p H$ dependence, Molecular dynamics simulations

\section{INTRODUCTION}

Enzymatic activity is usually pH-dependent, and this behavior is one of the standard parameters measured during in vitro kinetics studies of enzymes to identify catalytic residues and mechanisms. This factor has a more fundamental importance in biology, where an enzyme's biological activity is affected by the $\mathrm{pH}$ of its microenvironment. This $\mathrm{pH}$ may or may not correspond to the optimum found in laboratory kinetics studies and can vary depending on the organism, within different compartments of the cell or cell type, and is different in healthy cells compared to the more acidic conditions within tumor microenvironments. In the protein tyrosine phosphatase (PTP) family, $\mathrm{pH}$ dependency is typically bell-shaped, with an optimum ranging from 4.5 for TK-PTP, found in the hyperthermophilic archaeon Thermococcus kodakarensis, ${ }^{1}$ to 7.5 for PTP gamma, a mammalian PTP implicated in human tumor suppression. ${ }^{2}$ The variations in $\mathrm{pH}$ optima among PTPs likely reflects optimization for their biological roles.

The ubiquity of the PTP family in the biological kingdom reflects the fundamental role played by protein phosphorylation as a regulatory mechanism. The large family of diverse
PTPs, together with protein tyrosine kinases, modulates the phosphorylation level of tyrosine residues in the cell. The classical PTPs hydrolyze only phosphotyrosine (pTyr) residues of polypeptide substrates, while the dual-specific subfamily members (DSPs) also dephosphorylate phosphoserine (pSer) and phosphothreonine (pThr) residues. ${ }^{3-6}$ All PTPs share the conserved signature motif $\mathrm{HCX}_{5} \mathrm{R}(\mathrm{S} / \mathrm{T})$ forming the P-loop, which includes the nucleophilic cysteine and a conserved arginine, which, along with backbone amides, binds substrate and provides transition-state stabilization. PTPs follow a twostep catalytic mechanism with a cysteinyl-phosphate intermediate, shown in Figure 1. Many PTPs exhibit burst kinetics at $\mathrm{pH} \sim 6$, indicating that the first chemical step is rapid and

Received: February 9, 2021

Published: April 23, 2021 

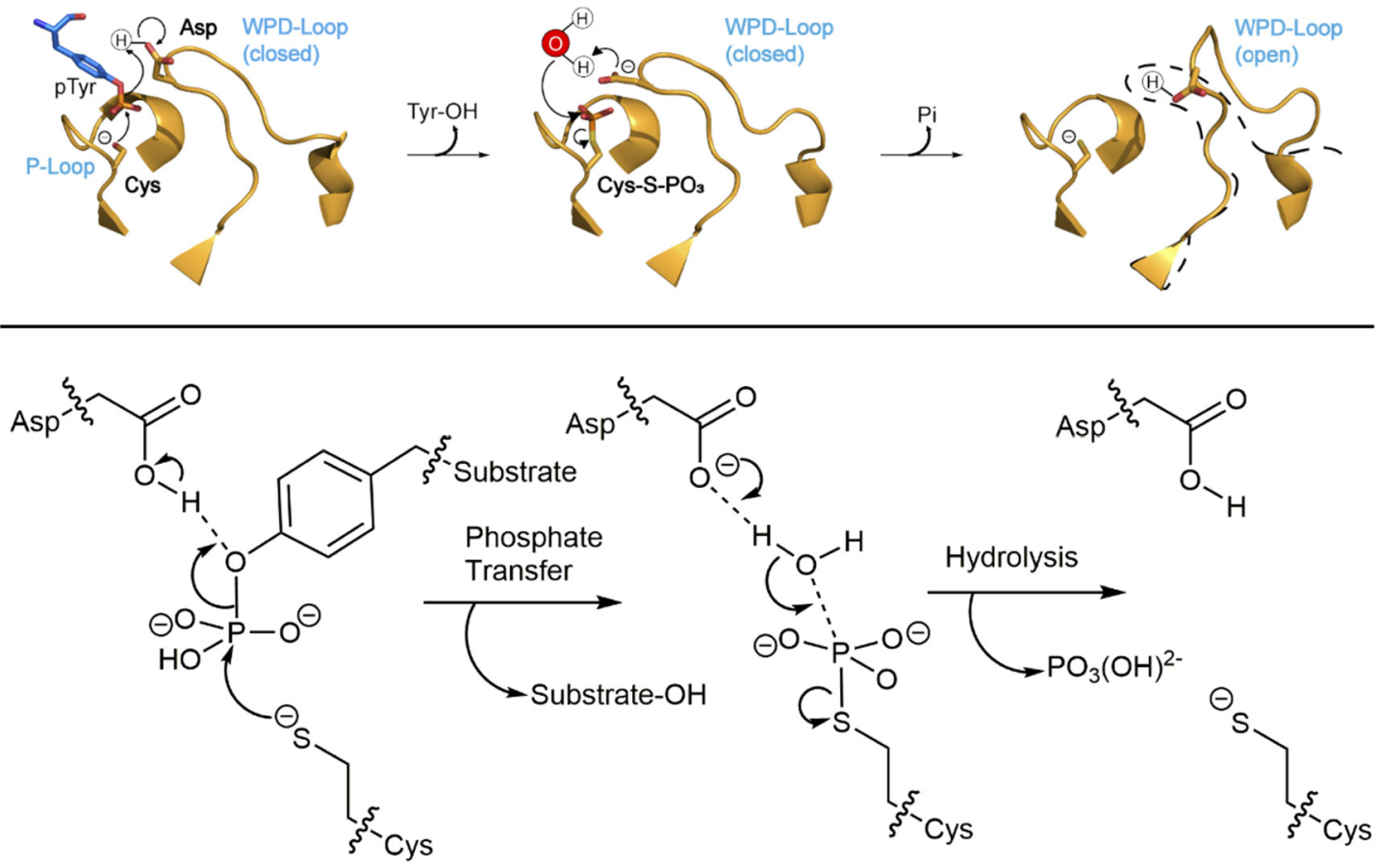

Figure 1. Mechanism of the PTP-catalyzed reaction, showing the mobile WPD-loop, the phosphate-binding P-loop, as well as the substrate, nucleophilic water molecule, and key catalytic side chains. Motion of the protein loop bearing the conserved general acid (i.e., the side chains of residues D356 in YopH and D181 in PTP1B) brings it into position to function as a general acid in the first step, where it protonates the leaving group during formation of a phosphocysteine intermediate. In the second step, the carboxylate form activates a nucleophilic water molecule.

the overall rate-determining step for $k_{\text {cat }}$ is the hydrolysis of the intermediate under these conditions.

In addition to the P-loop, PTPs share an aspartic acid that protonates the leaving group in the first chemical step and then, as a carboxylate, activates the nucleophilic water molecule in the second step. In the classical PTPs, this residue is found on a flexible loop of about a dozen residues of variable sequence with the exception of a highly conserved WPD sequence (Figure S1), leading to its designation as the WPDloop. X-ray studies show a high degree of structural similarity in the P-loops and in the WPD-loops; the latter are usually, but not exclusively, found in the catalytically competent, closed position in PTP complexes with oxyanions in the active site $^{10-14}$ and in a nonfunctional open position in ligand-free structures. ${ }^{15,16}$ Closure of the WPD-loop moves the conserved Asp residue about $9 \AA$ into the position needed for catalysis (Figure S2). Despite structural similarities, catalytic rates vary over several orders of magnitude within the PTP family, even among the classic PTPs. In this context, NMR experiments have revealed a correlation between WPD-loop dynamics and substrate cleavage rate in $\mathrm{YopH}$ and $\mathrm{PTP} 1 \mathrm{~B},{ }^{17}$ and recent computational work similarly suggests that turnover rates by these enzymes are modulated by the conformational dynamics of the WPD-loops of these enzymes. ${ }^{18}$

Following from this, the active-site Cys nucleophile (Figure 1) must be deprotonated for catalysis, and the $\mathrm{p} K_{\mathrm{a}}$ of this residue is significantly decreased from the typical solution value by the neighboring His residue, a conserved part of the signature P-loop motif. This His residue hydrogen bonds to the thiolate form of the Cys side chain, stabilizing the conjugate base form, and its mutation to Ala in $\mathrm{YopH}$ raises the Cys $\mathrm{p} K_{\mathrm{a}}$ from 4.67 to 7.35 . $^{7}$ The Cys residue is found at the bottom of the active site in contrast to the other conserved catalytic residue, the general acid, which is in the solvent- exposed WPD-loop. This requirement for a deprotonated Cys and a protonated Asp gives rise to bell-shaped $\mathrm{pH}$-rate profiles that are found for PTPs. ${ }^{19}$ However, despite the conservation of these two catalytic residues, the $\mathrm{pH}$-rate profiles of some PTPs are notably broader than others, in addition to the aforementioned variation in the $\mathrm{pH}$ of optimal catalysis. For example, YopH has a narrower profile ${ }^{20}$ than PTP1B, and its maximal activity occurs over a narrower range.

However, the $\mathrm{p} K_{\mathrm{a}}$ values reflected in a $\mathrm{pH}$-rate profile extracted from a fit of the kinetic data to the appropriate equation reflecting the number of ionizable catalytic groups are often distorted from the thermodynamic or "true" $\mathrm{p} K_{\mathrm{a}}$ of those groups. ${ }^{21-23}$ True $\mathrm{pK}$ a values are reflected in the $\mathrm{pH}$-rate profile only when a number of conditions are met. These include the condition of a single ionization state of the active site contributing to catalysis and when all prototropic equilibria (i.e., equilibria involving proton transfer processes) and other equilibria involving catalytic groups are fast with respect to the chemical steps. For example, substrate binding or dissociation that is not rapid relative to chemistry (referred to as sticky substrates) pushes the observed $\mathrm{p} K_{\mathrm{a}}$ values outward from their true values. ${ }^{24}$ The two chemical steps of the PTP mechanism utilize different ionization states, so the rate-determining step will change with $\mathrm{pH}$. Such a condition is known to affect the $\mathrm{p} K_{\mathrm{a}}$ values observed in $\mathrm{pH}$-rate profiles, one example being dihydrofolate reductase, where an active-site residue of $\mathrm{p} K_{\mathrm{a}} 6.5$ is observed at an apparent $\mathrm{p} K_{\mathrm{a}}$ of $8.4 .^{25}$ The correlation between protein motions and the rate of catalysis in the PTP family ${ }^{17}$ adds an additional complicating factor for this enzyme family. Specifically, contributions from WPD-loop dynamics to the chemical steps and how the rates of such motions vary with $\mathrm{pH}$ will affect the $\mathrm{pH}$-rate profile and thus the apparent or "kinetic" $\mathrm{p} K_{\mathrm{a}}$ values. 
Scheme 1. Kinetic Scheme for Conversion of Substrate to Product and Release, Including Equilibria of the WPD-Loop ${ }^{a}$

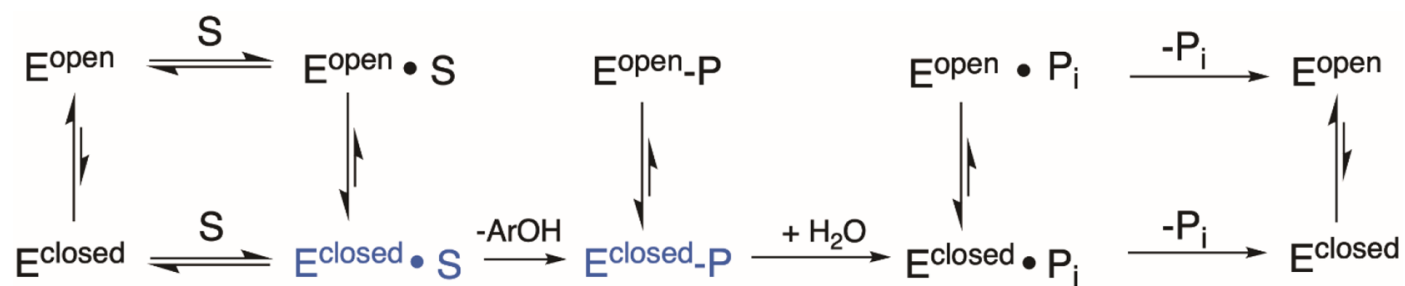

${ }^{a}$ Prototropic equilibria of the catalytic residues have been omitted. The catalytically functional species are shown in blue. Substrate can bind and product can be released from either loop-open or -closed forms, but the chemical steps can only occur from loop-closed conformations. Any factor that alters equilibria between open and closed forms may affect the extent to which the first or second step is rate-limiting at a given $\mathrm{pH}$, affecting $\mathrm{p} K_{\mathrm{a}}$ values observed in a $\mathrm{pH}$-rate profile.

Scheme 1 shows a simplified kinetic scheme for PTP catalysis including WPD-loop-open and -closed conformations. Because the loop moves the general acid into the active site from the side, rather than over the top of the active site, the substrate can bind to either the closed or open forms of the WPD-loop. However, substrate binding shifts the equilibrium from favoring the loop-open form to favoring the closed form, and only the loop-closed form can undergo catalysis in either of the chemical steps, highlighted in blue in Scheme 1. Loop equilibria affect the relative populations of the catalytically competent Michaelis complex and the phosphoenzyme intermediate. The $\mathrm{pH}$ will likely affect this equilibrium, as well as directly control the degree to which the first or second chemical step is rate-limiting. Both of these factors, in turn, perturb the apparent $\mathrm{p} K_{\mathrm{a}}$ values obtained from kinetic measurements from their thermodynamic values.

The kinetic $\mathrm{p} K_{\mathrm{a}}$ values, manifested in the catalytic reaction as a function of $\mathrm{pH}$ and which can differ from the thermodynamic ones, ${ }^{21-23}$ describe the biological activity of an enzyme in a particular environment. The divergence between kinetic $\mathrm{p} K_{\mathrm{a}}$ values and the true thermodynamic $\mathrm{p} K_{\mathrm{a}}$ values of catalytic groups provides a means for nature to tune the $\mathrm{pH}$ dependency of enzymatic activity that complements the well-established means by which proteins can alter the inherent thermodynamic $\mathrm{p} K_{\mathrm{a}}$ values of catalytic groups by modifying their electrostatic environment. ${ }^{26}$ In the PTP family, in particular, alterations in residues that affect protein motions that are connected to catalysis, particularly those of the WPDloop, have the potential to alter the $\mathrm{pH}$ dependency of the reaction without changes to interactions to either the nucleophilic Cys residue at the bottom of the active site or the conserved aspartic acid on the mobile WPD-loop (Figure 1).

As proof of this concept, we have identified a mutation to a noncatalytic residue on the WPD-loop of YopH that significantly increases its activity at low $\mathrm{pH}$. The resulting broadening of the $\mathrm{pH}$ dependency of catalysis of the G352T variant bestows more than an order of magnitude faster turnover at $\mathrm{pH} 4$, with no loss of activity at its $\mathrm{pH}$ optimum compared to the wild type (WT). Structural characterizations and computational modeling show how this mutation shifts the equilibrium between the WPD-loop-open and -closed states. The kinetic $\mathrm{p} K_{\mathrm{a}}$ of the Cys residue responsible for the lower limb of the $\mathrm{pH}$-rate profile is lowered in G352T by nearly a full unit, while titration of the active-site Cys residue shows only a small change in its thermodynamic $\mathrm{p} K_{\mathrm{a}}$. Interestingly, the corresponding $\mathrm{T} 177 \mathrm{G}$ variant of PTP1B shows a more modest effect and in the opposite direction. The documented correlation between WPD-loop dynamics and catalysis in YopH and $\mathrm{PTP}_{1 \mathrm{~B}}{ }^{17,18}$ provides a rationale for the altered $\mathrm{pH}$ dependencies of these variants.

\section{MATERIALS AND METHODS}

\section{Chemicals}

Dithiothreitol (DTT) and ampicillin (AMP) were purchased from GoldBio. Restriction enzymes were purchased from Integrated DNA Technologies. Protease-inhibitor tablets were purchased from SigmaAldrich. All other buffers and reagents were purchased from SigmaAldrich or Fisher. The substrates $p$-nitrophenyl phosphate $(p N P P)^{27}$ and $p$-nitrophenyl phosphorothioate ( $p$ NPPS $)^{28}$ were synthesized using published methods. Crystallography screens, trays, and coverslips were purchased from Hampton Research.

\section{Mutagenesis and Expression}

The plasmid pEt-19b encoding wild-type human protein PTP1B was provided by Dr. N. K. Tonks, and the plasmid pEt-43.1b (+) encoding wild-type Yersinia PTP was provided by Dr. Z.-Y. Zhang.

PTP1B T177G was made by substituting residue T177 of wild-type PTP1B via the Q5-SDM kit (New England Biolabs) with G from the YopH WPD-loop in the corresponding region (Table S1). YopH G352T was made by the same method, replacing residue G352 of wild-type YopH with $\mathrm{T}$ from PTP1B in the corresponding region. Both point mutants were created using polymerase chain reaction (PCR) with primers encoding residues before the mutation and the target mutation itself. The mutant DNA was then cleaved using the restriction enzyme DpnI and ligated into either the pEt-19b or pEt43.1b (+) vector using T4 ligase. The primers used are listed in Table S2.

The DNA was transformed into BL21-DE3 cells and grown overnight at $37^{\circ} \mathrm{C}$ on an LB culture plate containing $10 \%$ ampicillin. One colony was selected and placed into $10 \mathrm{~mL}$ of SOC media containing $10 \%$ ampicillin and grown overnight. The following morning, $1 \mathrm{~L}$ of LB media containing $10 \%$ ampicillin was inoculated with the $10 \mathrm{~mL}$ of overnight growth and shaken at $170 \mathrm{rpm}$ at $37^{\circ} \mathrm{C}$ until the $\mathrm{OD}_{600}$ reached 0.6-0.8. After the optimal $\mathrm{OD}$ was reached, the $1 \mathrm{~L}$ growth was induced by $0.1 \mathrm{mM}$ isopropyl $\beta$-D-thiogalactoside (IPTG) and shaken at $170 \mathrm{rpm}$ at room temperature overnight. The cells were harvested by centrifugation at $12,000 \mathrm{~g}$ for $30 \mathrm{~min}$ at $4{ }^{\circ} \mathrm{C}$ and stored at $-80{ }^{\circ} \mathrm{C}$.

\section{Protein Purification}

Both mutants were expressed and purified as for the wild-type parent PTPs. ${ }^{19,29}$ PTP1B T177G cells were thawed on ice and resuspended in $10 \times$ their equivalent volume of a lysis buffer, consisting of $50 \mathrm{mM}$ imidazole pH 7.5, $1 \mathrm{mM}$ EDTA, $3 \mathrm{mM}$ DTT, and 10\% glycerol with $0.5 \mathrm{mg} / \mathrm{mL}$ aprotinin, $0.7 \mathrm{mg} / \mathrm{mL}$ pepstatin, and $0.5 \mathrm{mg} / \mathrm{mL}$ leupeptin. YopH cells were thawed and resuspended in $100 \mathrm{mM}$ acetate $\mathrm{pH}$ 5.7, $100 \mathrm{mM} \mathrm{NaCl}, 1 \mathrm{mM}$ EDTA, $3 \mathrm{mM}$ DTT, and $10 \%$ glycerol with the same protease inhibitors. The cells were lysed by sonication at $60 \%$ power for 10 pulses and then mixed on ice for 1 min and repeated 4-6 times until completely lysed. The cell lysate 
Table 1. Data Collection and Refinement Statistics

\begin{tabular}{|c|c|c|c|c|}
\hline & PTP1B T177G (ligand-free) & PTP1B T177G $\left(\mathrm{VO}_{4}\right)$ & YopH G352T (ligand-free) & YopH G352T $\left(\mathrm{VO}_{4}\right)$ \\
\hline PBD ID & 7L0C & $7 \mathrm{LOH}$ & 7L0I & 7L0M \\
\hline \multicolumn{5}{|l|}{ Data Collection } \\
\hline source & SSRL 9-2 & SSRL 9-2 & SSRL 9-2 & SSRL 9-2 \\
\hline space group & $P 3_{1} 21$ & $P 3_{1} 21$ & $P 2_{1} 2_{1} 2_{1}$ & $P 2_{1} 2_{1} 2_{1}$ \\
\hline \multicolumn{5}{|l|}{ Cell dimensions } \\
\hline$a, b, c(\AA)$ & $88.506,88.506,104.432$ & $88.471,88.471,104.979$ & $49.183,56.351,98.513$ & $49.231,56.091,98.302$ \\
\hline$\alpha, \beta, \gamma(\mathrm{deg})$ & $90,90,120$ & $90,90,120$ & $90,90,90$ & $90,90,90$ \\
\hline \multirow[t]{2}{*}{ resolution $(\AA)$} & $43.2-1.80$ & $43.3-2.10$ & $49.2-2.02$ & $49.2-2.00$ \\
\hline & $(1.86-1.80)$ & $(2.18-2.10)$ & $(2.10-2.02)$ & $(2.10-2.00)$ \\
\hline $\mathrm{CC}_{1 / 2}$ & 0.699 & 0.632 & 0.467 & 0.796 \\
\hline$I / \sigma I$ & $31.5(0.9)$ & $29.8(1.3)$ & $22.2(14.5)$ & $88.1(8.6)$ \\
\hline completeness (\%) & $99.9(100.0)$ & $100.0(100.0)$ & $94.3(79.6)$ & $95.7(81.0)$ \\
\hline redundancy & $18.9(14.8)$ & $18.6(14.1)$ & $5.5(3.0)$ & $7.7(5.0)$ \\
\hline no. reflections & $44,193(4324)$ & $28,286(2773)$ & $17,623(1437)$ & $18,269(1520)$ \\
\hline \multicolumn{5}{|l|}{$\underline{\text { Refinement }}$} \\
\hline$R_{\text {work }} / R_{\text {free }}$ & $0.163 / 0.175$ & $0.178 / 0.208$ & $0.202 / 0.253$ & $0.178 / 0.226$ \\
\hline \multicolumn{5}{|l|}{ no. atoms } \\
\hline protein & 2390 & 2425 & 2117 & 2153 \\
\hline ligand/ion & 0 & 5 & 15 & 5 \\
\hline water & 293 & 147 & 148 & 145 \\
\hline \multicolumn{5}{|l|}{$\beta$-factors } \\
\hline protein & 28.9 & 49.5 & 34.9 & 32.3 \\
\hline ligand/ion & & 39.4 & 42.0 & 28.2 \\
\hline solvent & 39.8 & 49.9 & 35.8 & 35.7 \\
\hline \multicolumn{5}{|l|}{ rms deviations } \\
\hline bond lengths $(\AA)$ & 0.007 & 0.009 & 0.007 & 0.008 \\
\hline bond angles (deg) & 0.97 & 1.17 & 0.93 & 1.11 \\
\hline \multicolumn{5}{|l|}{ Ramachandran } \\
\hline favored (\%) & 97.3 & 97.6 & 96.4 & 96.4 \\
\hline allowed (\%) & 2.4 & 2.0 & 3.2 & 3.2 \\
\hline outliers (\%) & 0.3 & 0.3 & 0.4 & 0.4 \\
\hline
\end{tabular}

was centrifuged at $4{ }^{\circ} \mathrm{C}$ at $29000 \mathrm{~g}$ for $30 \mathrm{~min}$. The supernatant was filtered with a $0.45 \mu \mathrm{m}$ syringe filter.

The filtrate was then purified via a $5 \mathrm{~mL}$ HiTrap Q HP column attached above a $5 \mathrm{~mL}$ HiTrap SP HP column using an FPLC filtration system. Both columns were equilibrated with lysis buffer. The cell lysate was loaded onto the columns at $1.5 \mathrm{~mL} / \mathrm{min}$; the HiTrap Q HP column was removed after loading, and the HiTrap SP $\mathrm{HP}$ column was washed with lysis buffer until the absorbance at 280 $\mathrm{nm}$ baselined. Elution for PTP1B T177G was processed using a 100\% gradient with elution buffer containing $500 \mathrm{mM} \mathrm{NaCl}, 50 \mathrm{mM}$ imidazole pH 7.5, $1 \mathrm{mM}$ EDTA, $3 \mathrm{mM}$ DTT, and 10\% glycerol, and YopH with $100 \mathrm{mM}$ acetate $\mathrm{pH} 5.7,500 \mathrm{mM} \mathrm{NaCl}, 1 \mathrm{mM}$ EDTA, 3 $\mathrm{mM}$ DTT, and $10 \%$ glycerol. Eluted fractions exhibiting absorbance at $280 \mathrm{~nm}$ were collected and tested with $p$ NPP for phosphatase activity. Fractions that showed activity were assayed for purity on a $15 \%$ SDSPAGE gel.

The active fractions were pooled (ranging from 30 to $40 \mathrm{~mL}$ ) and concentrated to $<12 \mathrm{~mL}$, loaded onto a pre-equilibrated HiLoad 26/ 60 Superdex 200 prepgrade column (GE), and purified using $10 \mathrm{mM}$ Tris buffer $\mathrm{pH} 7.5$, with $25 \mathrm{mM} \mathrm{NaCl}, 0.2 \mathrm{mM}$ EDTA, and $3 \mathrm{mM}$ DTT for PTP1B T177G, and $100 \mathrm{mM}$ acetate $\mathrm{pH} 5.7,100 \mathrm{mM} \mathrm{NaCl}$, $1 \mathrm{mM}$ EDTA, $3 \mathrm{mM}$ DTT for YopH G352T. Fractions were assayed with $p$ NPP for activity and purity on a $15 \%$ SDS-PAGE gel. Pure protein was concentrated to $10-35 \mathrm{mg} / \mathrm{mL}$ and either kept on ice to immediately set up crystal trays or diluted with $10 \%$ glycerol and frozen with liquid nitrogen and stored at $-80{ }^{\circ} \mathrm{C}$ in aliquots.

\section{X-ray Crystallography}

Crystals for PTP1B T177G were grown by hanging drop vapor diffusion using $15 \mathrm{mg} / \mathrm{mL}$ protein and a well solution of $0.1 \mathrm{M}$ Tris hydrochloride $\mathrm{pH} 7.5-8.5,0.2 \mathrm{M}$ magnesium acetate tetrahydrate, and $20-25 \%$ PEG 8000 at a 2:2:0.5 protein/well/20\% benzamidine hydrochloride drop ratio. The vanadate-bound structures were obtained by adding $5 \mathrm{mM}$ sodium metavanadate $\left(\mathrm{Na}_{3} \mathrm{VO}_{4}\right)$ to the protein for cocrystallization. Crystals were transferred to a cryoprotectant solution containing mother liquor, 20\% benzamidine hydrochloride, and $50 \%$ sucrose before flash freezing in liquid nitrogen.

Crystals for YopH G352T were grown by hanging drop vapor diffusion using $2 \mathrm{mg} / \mathrm{mL}$ protein and a well solution of $0.1 \mathrm{M}$ HEPES $\mathrm{pH} 7.5$ and $15-34 \%$ PEG 3350 at a $2: 2$ protein/well drop ratio; 0.5 $\mu \mathrm{L}$ seeding stock was then added to the drop. The microcrystals were generated by crushing $1 \mu \mathrm{L}$ of PTP1B T177G crystals in the well solution and diluted to $1: 10^{8}$ ratio. The vanadate-bound structures were obtained by adding $5 \mathrm{mM}$ sodium metavanadate $\left(\mathrm{Na}_{3} \mathrm{VO}_{4}\right)$ to the protein for cocrystallization. Crystals were transferred to a cryoprotectant solution containing mother liquor with $5 \mathrm{mM}$ sodium metavanadate and $20 \%$ glycerol before being flash frozen in liquid nitrogen.

Diffraction data were collected on Stanford Radiation Lightsource (SSRL) beamline 9-2 (Table 1). Molecular replacement was performed with Phaser-MR ${ }^{30}$ as implemented in Phenix ${ }^{31}$ using WT PTP1B (PDB ID: $3180^{10}$ ) and YopH W354Y (PDB ID: 4YAA ${ }^{32}$ ) as search models for PTP1B T177G and YopH G352T, respectively. Phenix.refine ${ }^{30}$ was used for refinement. Model building was performed using Coot. ${ }^{33}$ All figures of the enzyme structures and structural alignments therein were made using Pymol (The PyMOL Molecular Graphics System, version 1.2r3pre, Schrödinger, LLC).

\section{Steady-State Kinetics}

Steady-state kinetic parameters were measured at $25{ }^{\circ} \mathrm{C}$. Concentrated protein aliquots were thawed on ice and diluted with a buffer base mix (BBM) containing $50 \mathrm{mM}$ sodium acetate, $100 \mathrm{mM}$ Tris, and $100 \mathrm{mM}$ bis-Tris from $\mathrm{pH} 4.0$ to $\mathrm{pH}$ 7.5. This buffer system 


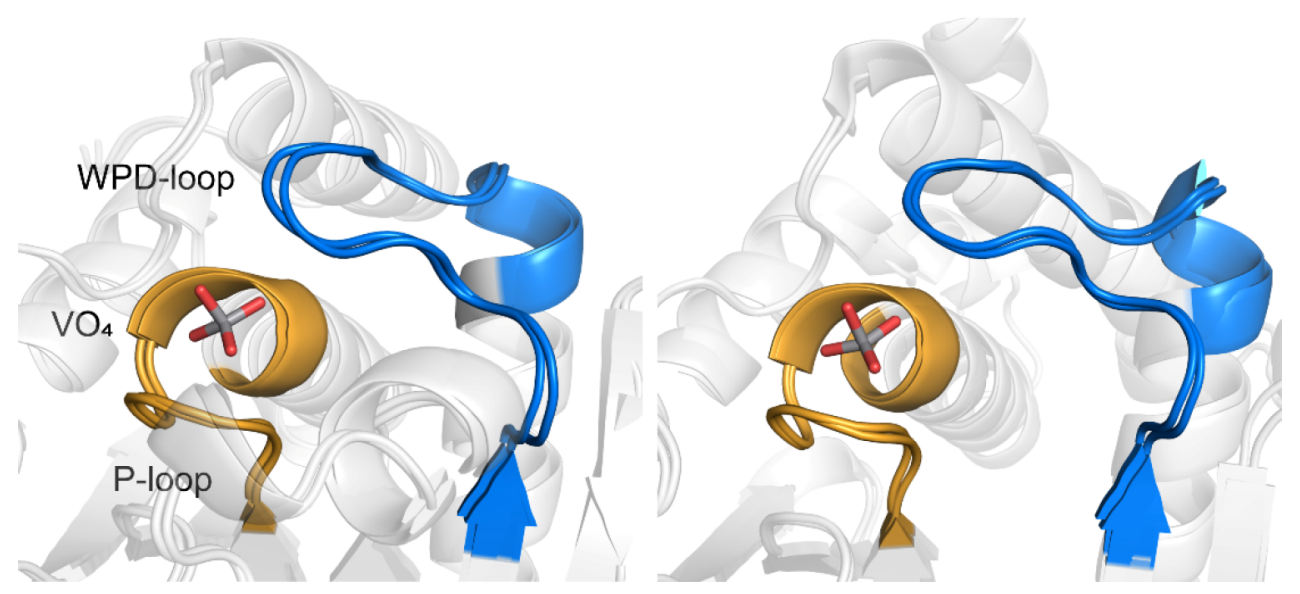

Figure 2. Comparison of active-site structures of ligand-bound mutant PTPs match well with those for their wild-type counterparts. The remainder of the scaffold is virtually identical between the liganded and ligand-free forms of each enzyme (backbone RMSD of 0.461 and 0.233 A for PTP1B and YopH, respectively). Left: Vanadate-bound WT YopH (PDB ID: $2 \mathrm{I}_{42}{ }^{40}$ ) and vanadate-bound G352T YopH (this work). Right: vanadatebound WT PTP1B (PDB ID: 3I80 ${ }^{10}$ ) and vanadate-bound T177G PTP1B (this work). Ligand-bound WPD-loops are colored in blue, and ligandbound P-loops are colored in yellow.

maintains constant ionic strength throughout the $\mathrm{pH}$ range examined. A $50 \mathrm{mM}$ solution of the dicyclohexylammonium salt of $p$ NPP was prepared in the buffer base mix. The reactions were run on a 96-well plate using diluted enzyme protein concentrations from 0.0084 to $0.0106 \mu \mathrm{M}$ (PTP1B T177G) or 0.0004-0.0007 $\mu \mathrm{M}$ (YopH G352T) and substrate concentrations from 0.76 to $22.73 \mathrm{mM}$. Reactions were allowed to proceed for $2 \mathrm{~min}$ for PTP1B T177G and $3 \mathrm{~min}$ for YopH G352T. The reactions were quenched using $50 \mu \mathrm{L}$ of $5 \mathrm{M} \mathrm{NaOH}$, and the amount of the product $p$-nitrophenol was assayed from the absorption at $400 \mathrm{~nm}$ using the molar extinction coefficient of 18,300 $\mathrm{M}^{-1} \mathrm{~cm}^{-1}$. Reaction blanks were made using identical conditions replacing the enzyme with buffer to correct for non-enzymatic hydrolysis of the substrate. The amount of product released and elapsed time were used to calculate the initial rates. The data were fitted to the Michaelis-Menten equation to obtain steady-state kinetic parameters. Kinetic data were obtained on both mutants as a function of $\mathrm{pH}$ to obtain $\mathrm{pH}$-rate profiles. The bell-shaped $\mathrm{pH}$-rate profiles were fitted to eq 1 , the standard equation relating the dependence of the observed $k_{\text {cat }}$ to the maximal, or limiting, value as a function of $\mathrm{pH}$, where catalysis is dependent on two ionizable residues, one protonated and the other deprotonated. ${ }^{34}$

$$
k_{\mathrm{cat}}=\frac{k_{\mathrm{cat}}^{\lim }}{\left(1+\frac{\left[\mathrm{H}^{+}\right]}{K_{1}}+\frac{\left[K_{2}\right]}{\left[\mathrm{H}^{+}\right]}\right)}
$$

\section{Active-Site Cysteine Thiol Titrations}

Enzyme stock was diluted in buffer base mix solution (100 mM sodium acetate, $50 \mathrm{mM}$ bis-Tris, $50 \mathrm{Mm}$ Tris- $\mathrm{HCl}$ ) at various $\mathrm{pH}$ values with 1-10 mM iodoacetate. Reaction blank was made with the same reagents omitting the iodoacetate. Enzyme was incubated with iodoacetate and an aliquot was removed at 5-10 min intervals to assay for activity with $3.8 \mathrm{mM} p \mathrm{NPP}$. Phosphatase activity assays were allowed to proceed for $180 \mathrm{~s}$ in $\mathrm{BBM}$ at $\mathrm{pH} 5.0$ and quenched with 5 $\mathrm{M} \mathrm{NaOH}$. Formation of $p$-nitrophenol product was assayed as described above. Experiments were done at $0.5 \mathrm{pH}$ unit intervals from $\mathrm{pH} 4.0$ to $\mathrm{pH} 7.0$.

At each $\mathrm{pH}$, residual activities as a function of time were plotted to obtain $k_{\mathrm{obs}}\left(\mathrm{min}^{-1}\right)$ for the inactivation rate constant at each iodoacetate concentration. These $k_{\mathrm{obs}}$ values were plotted against iodoacetate concentrations to obtain the second-order rate constant $k_{2}\left(\mathrm{M}^{-1} \mathrm{~min}^{-1}\right)$ as a function of $\mathrm{pH}$. The $\mathrm{pH}$ dependency of iodoacetate inactivation was obtained by plotting $k_{2}$ as a function of
$\mathrm{pH}$ (Figure S4) and fitted to eq 2 to obtain the thermodynamic $\mathrm{p} K_{\mathrm{a}}$ value for the active-site cysteine thiol. The same methodology has been used to obtain the cystine $\mathrm{p} K_{\mathrm{a}}$ values in $\mathrm{YopH}^{7}$ and VHR. ${ }^{35}$

$$
k_{\text {inactivation }}=\frac{k_{\text {inactivation }}^{\lim }}{\left(1+\frac{\left[\mathrm{H}^{+}\right]}{K_{1}}\right)}
$$

\section{Molecular Dynamics Simulations and Analysis}

Molecular dynamics (MD) simulations were performed using the AMBER $18^{36}$ simulation package with the $\mathrm{ff} 14 \mathrm{SB}^{37}$ force field and TIP3P ${ }^{38}$ water model to describe protein atoms and water molecules, respectively. Simulations of both WT PTPs and their corresponding point mutants were initiated where possible from either the corresponding X-ray crystal structure obtained from this study or structures used in previous studies (see Table S3 for all structures used). ${ }^{14,39,40}$ Simulations were performed on the ligand-free forms of each enzyme variant. For the open WPD-loop conformations of T177G and G352T point mutants for which no crystal structures exist, the WT crystal structures (with substitutions made using the Dunbrack rotamer library ${ }^{41}$ as implemented in PyMOL, selecting the side chain rotamer that minimizes steric clashes with the surroundings) were used as the starting structure for the MD simulations. MolProbity ${ }^{42}$ was used to identify any necessary Asn, Gln, and His rotamer changes, whereas PROPKA v.3.1 $1^{43}$ was used to assign protonation states to simulate at an effective $\mathrm{pH}$ of 5 for all enzymes (see Table S4 for all residues assignments), with care taken to be consistent for simulations of the same PTP. Structures were solvated in an octahedral water box (keeping all crystallographic waters) such that all solute atoms were at least $10 \AA$ away from the box boundaries, and $\mathrm{Na}^{+}$or $\mathrm{Cl}^{-}$ions were added as necessary to ensure system neutrality.

We followed a standard minimization, heating, and equilibration protocol (described in full in the Supporting Information) to prepare each structure for production of MD simulations in the NPT ensemble $(300 \mathrm{~K}, 1 \mathrm{~atm})$. For each system, 10 replicas of $100 \mathrm{~ns}$ long MD simulations were performed starting from both the closed and open WPD-loop conformations. MD simulations were run with a $2 \mathrm{fs}$ time step (with the SHAKE $^{44}$ algorithm applied) and an $8 \AA$ direct space nonbonded cutoff, with long-range electrostatics evaluated using the particle-mesh Ewald ${ }^{45}$ method. Temperature and pressure were regulated using Langevin temperature control (collision frequency of $1 \mathrm{ps}^{-1}$ ) and a Berendsen barostat (pressure relaxation 


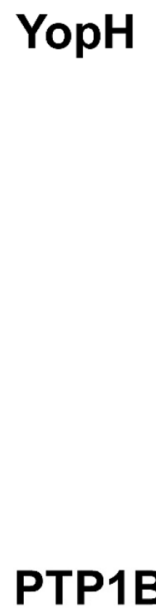

A

\section{Mutant}

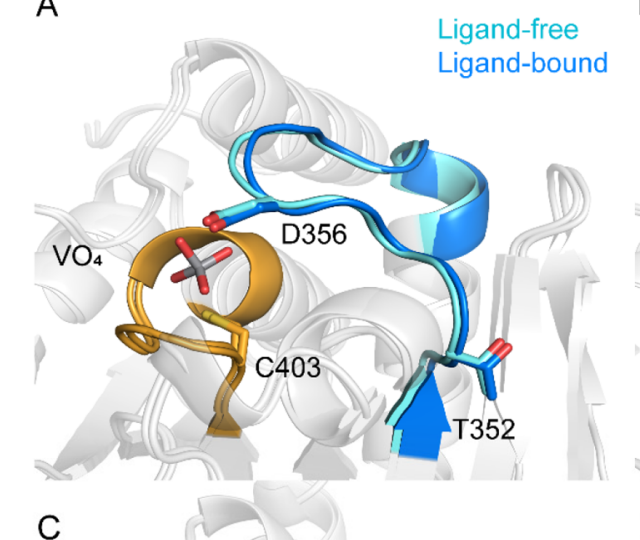

B

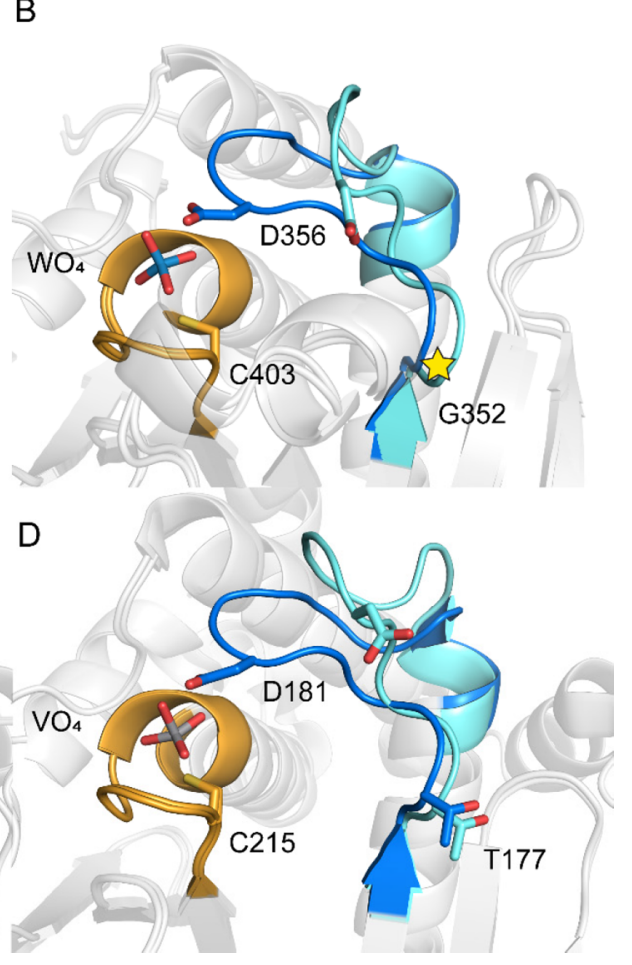

Figure 3. Active-site structures of mutant PTPs crystallize in the closed WPD-loop conformation in both ligand-free and ligand-bound conditions, indicating a shifted loop equilibrium. (A) Ligand-free and vanadate-bound G352T YopH (this work). (B) Ligand-free (loop open) (PDB ID: $1 \mathrm{YPT}^{14}$ ) and tungstate-bound (PDB ID: 1YTW ${ }^{12}$ ) (loop closed) complexes of WT YopH. (C) Ligand-free and vanadate-bound PTP1B T177G (this work). (D) Ligand-free WT PTP1B (PDB ID: $2 \mathrm{CM} 2^{15}$ ) and vanadate-bound WT PTP1B (PDB ID: 3 I $^{10} 0^{10}$ ). The WPD-loops in ligand-free enzymes are colored in cyan, ligand-bound WPD-loops are colored in blue, and P-loops are colored in yellow. Vanadate ions are only present in ligand-bound forms. The positions of G352 in YopH and G177 in PTP1B are marked by the star symbols.

time of $1 \mathrm{ps}$ ). Simulation analysis was performed using a combination of CPPTRAJ ${ }^{46}$ and the $\mathrm{R}$ software package ${ }^{47}$ (for statistical tests). Residues 176-190 and 351-565 of the WPD-loops of PTP1B and YopH, respectively, were used for the $\mathrm{C}_{\alpha}$ root mean square deviation (RMSD) calculations. Hydrogen bonds were defined as being present if the donor-acceptor distance was $\leq 3.5 \AA$ and if the donorhydrogen-acceptor angle was $180 \pm 45^{\circ}$.

\section{RESULTS AND DISCUSSION}

\section{X-ray Crystal Structures}

Structures were obtained of the PTP1B T177G and YopH G352T ligand-free enzymes and in complex with vanadate. Comparison of the variant structures with those of the native enzymes show no significant backbone perturbations in the WPD-loop regions or elsewhere (Figure 2).

In both ligand-free structures, the WPD-loops are observed in the closed position (Figure 3). This is in contrast to the typical observation of this loop in the open, noncatalytic position in crystal structures of these and other PTPs lacking a bound oxyanion. There has been no X-ray structure reported of ligand-free WT YopH with its loop in the closed conformation. There is an example of such a structure of PTP1B; ${ }^{48}$ however, this structure (PDB ID: 1 SUG $^{48}$ ) was subsequently re-refined and found to contain electron densities indicating the presence of both open and closed WPD-loop states. ${ }^{39}$ In contrast, we do not observe any electron density for the open state in the crystal structure of PTP1B T177G. Particularly, for the YopH variant, the closed conformation found suggests the G352T mutation has shifted the equilibrium of the ligand-free enzyme to favor the closed conformation.

The G352T substitution in YopH introduces a new $3.3 \AA$ hydrogen bond between the side chain of T352 and the backbone carbonyl of M328 (Figure S3) in both the ligandfree and vanadate-bound structures, stabilizing the loop-closed conformation. If this hydrogen bond is only present, or is tighter, in the loop-closed conformation, it would rationalize the presence of this conformation in the ligand-free structure in contrast to the native enzyme. We were unable to obtain a crystal structure of a loop-open conformation of the mutant, and interactions of T352 in that conformation are unknown.

For the PTP1B variant, crystal structures do not provide a clear rationale for how the T177G mutation might affect loop equilibrium. Residue $\mathrm{T} 177$ is involved in a network of hydrogen bonds in native PTP1B. In the WPD-loop open conformation, the backbone amide of T177 hydrogen bonds both with the side chain of R112 and with the threonine hydroxyl of T177. In the loop-closed conformation, the interaction with R122 is lost and the internal hydrogen bond with the hydroxyl group has lengthened from 3.3 to $3.5 \AA$ (Figure 4). The loss of the R122 interaction results from the rotation of the guanidinium side chain and the resulting new hydrogen bonds to the bound substrate or oxyanion inhibitors in the loop-closed conformation. As shown in Figure 4, a comparison of ligand-free native PTP1B in WPD-loop-open and -closed states shows that the primary difference involving T177 is loss of the hydrogen bond between the backbone carbonyl of T177 and the guanidinium side chain of R122 in 


\section{Ligand-free}

WT PTP1B

\section{PTP1B T177G}

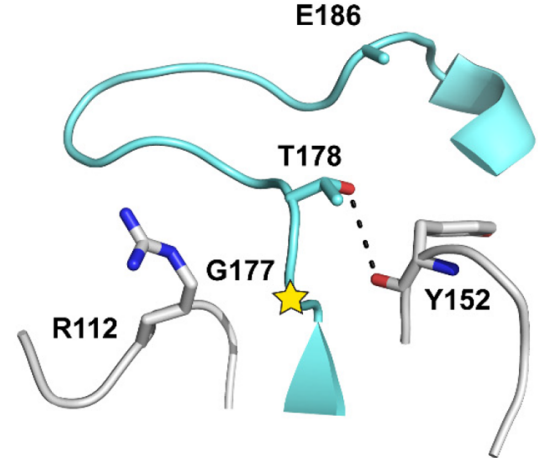

Ligand-bound
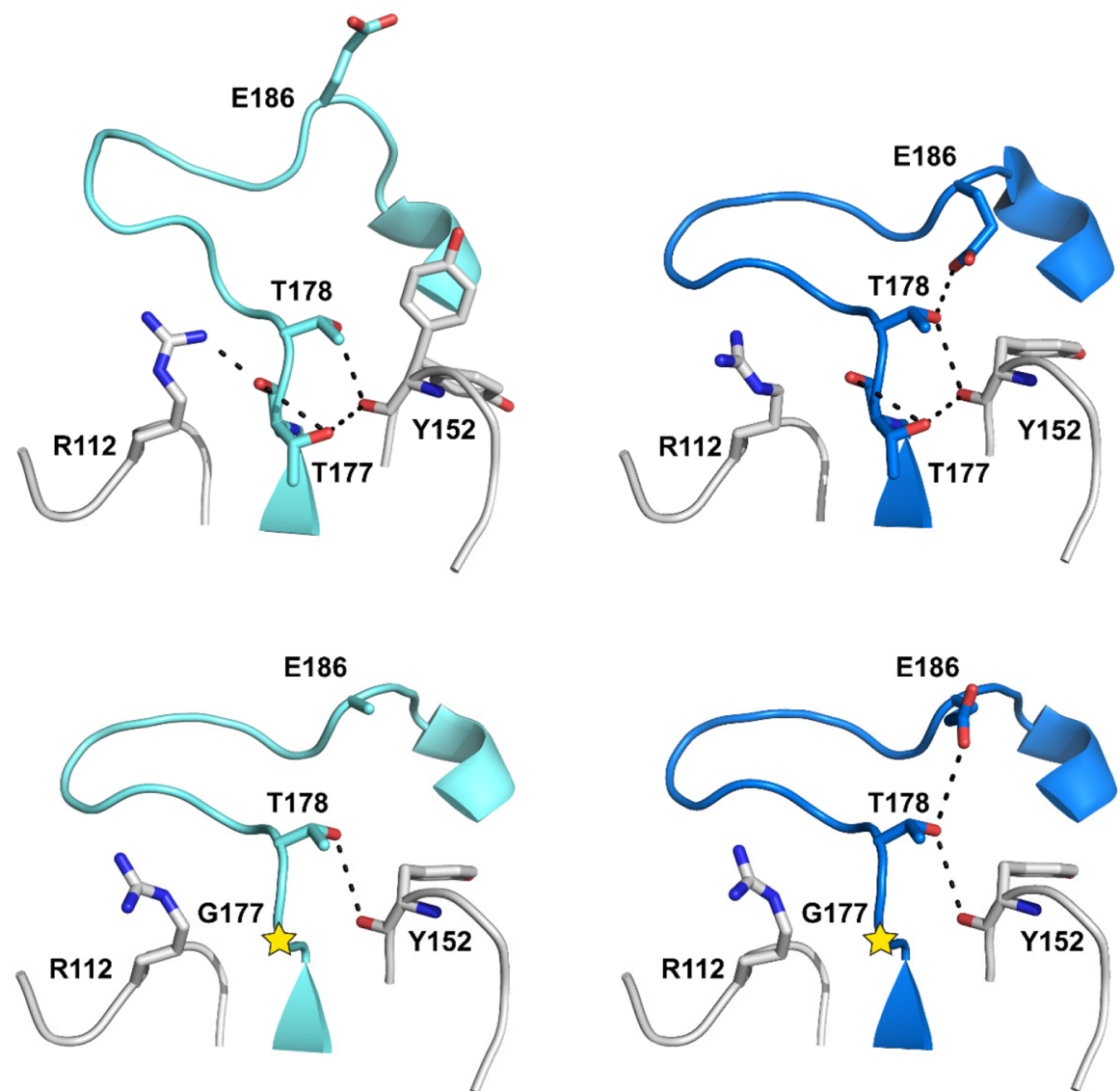

Figure 4. Interaction between R112 and T177 in loop open form of WT PTP1B is lost in the loop-closed form and in PTP1B T177G. The guanidinium side chain forms a hydrogen bond in the loop-open form, while the side chain rotates away from the WPD-loop in the closed form. The side chain conformation of E186 is uncertain in the crystal structure of ligand-free PTP1B T177G (this work). Vanadate-bound WT PTP1B (PDB ID: $3 I_{180^{10}}$ ) and ligand-free WT PTP1B (PDB ID: 2CM2 ${ }^{15}$ ) are previously reported structures. The positions of G177 in PTP1B structures are marked by the star symbols.

the closed state and a slight tightening of the two hydrogen bonds of the side chain hydroxyl.

In native PTP1B, the backbone amide carbonyl of Y152 is within the hydrogen bonding distance of the hydroxyl groups of T177 and T178 in both WPD-loop conformations. In this way, T177 and Y152 act as anchors to the interaction between T178 and E186 (Figure 4). In the loop-open conformation, the carboxylic acid group of E186 changes its direction from pointing toward the $\mathrm{N}$-terminal of the loop to away from T177; therefore, the polar interaction between these residues is not observed in the opened loop. In the mutant PTP1B structure, most of the interactions that stabilize the closed conformation remain intact; however, the polar interaction between the side chain of T177 and the backbone of Y152 is lost. In addition to the loss of this interaction between $\mathrm{T} 177$ and Y152, no other obvious changes in the X-ray crystal structures indicate alterations of the WPD-loop equilibria. This is consistent with the observation that the T177 side chain is part of a network of noncovalent interactions that are likely responsible for stabilizing the open conformation of the WPDloop, and therefore substitutions at position 177 would be expected to disrupt this interaction network, causing a population shift toward a closed conformation of the loop. ${ }^{49}$ We note that the observation of a closed loop in both ligand- free and bound forms does not infer that the WPD-loop has been rigidified and is no longer mobile. That is, retention of loop motion is supported by NMR experiments on the related PTP1B T177A variant, which showed that the loop still samples both closed and open conformations but with a shift in population to favor the closed conformation. ${ }^{49}$

\section{Kinetics}

Figure 5 shows the $\mathrm{pH}$ dependency of the turnover number with the substrate $p$ NPP for the native enzymes and variants. A modest change to the profile for PTP1B is observed in the T177G variant. The basic limb of the profile and the maximal turnover number are unaltered, while the acidic limb is shifted somewhat to higher $\mathrm{pH}$, with the net result that the T177G variant below $\mathrm{pH} 6$ is a slower enzyme than native PTP1B. An opposite and more dramatic effect occurs in YopH. The WT YopH kinetic $\mathrm{p} K_{\mathrm{a}}$ values (Table 2) are each $\sim 0.4$ units higher than those previously reported at a higher temperature and different buffer system. ${ }^{20}$ The difference between them is nearly identical to the previous report, and this smaller difference in YopH relative to PTP1B gives rise to its narrower $\mathrm{pH}$ profile. Its $\mathrm{G} 352 \mathrm{~T}$ variant shows a much broader $\mathrm{pH}$-rate profile than WT, with a kinetic $\mathrm{p} K_{\mathrm{a}}$ of the nucleophilic Cys residue lower by about a full unit (Table 2). The native YopH enzyme has a $\mathrm{pH}$ optimum lower than that of PTP1B, 5.5 


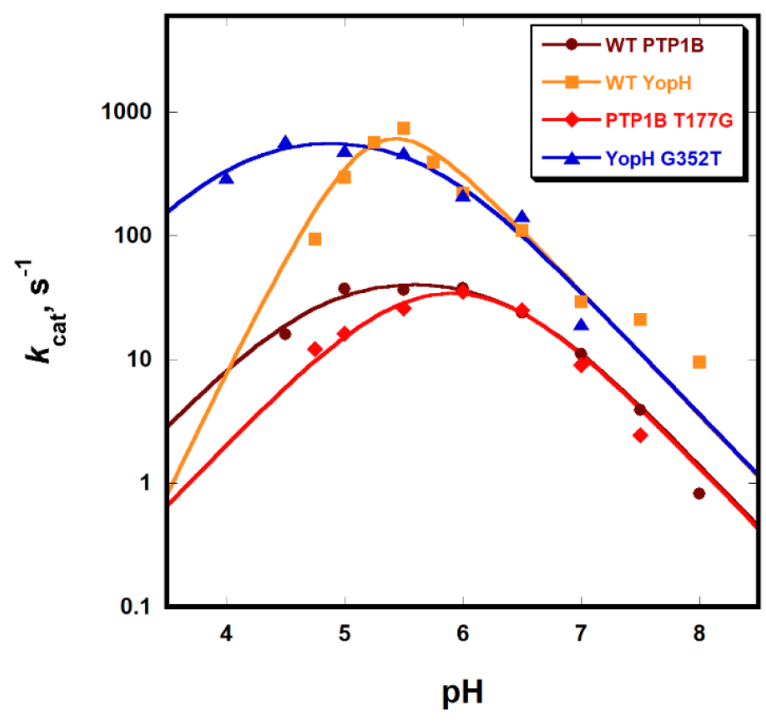

Figure 5. YopH G352T variant shows higher enzymatic activity at low $\mathrm{pH}$ than WT due to altered kinetic $\mathrm{p} K_{\mathrm{a}}$ values resulting in a broader pH-rate dependency. The orthogonal T177G PTP1B mutant shows more modest effects in the opposite direction, a slight narrowing of the profile, and lower activity at low $\mathrm{pH}$ compared to that with WT.

Table 2. Kinetic $p K_{\mathrm{a}}$ Values for Wild-Type Enzymes and Mutants Obtained from Fits of the $\mathrm{pH}$-Rate Data ${ }^{a}$

\begin{tabular}{lcc}
\multicolumn{1}{c}{ enzyme } & $\mathrm{p} K_{\mathrm{a} 1}$ & $\mathrm{p} K_{\mathrm{a} 2}$ \\
WT PTP1B & $5.08 \pm 0.20$ & $6.44 \pm 0.26$ \\
PTP1B T177G & $5.44 \pm 0.37$ & $6.37 \pm 0.28$ \\
WT YopH & $5.02 \pm 0.27$ & $5.58 \pm 0.34$ \\
YopH G352T & $4.06 \pm 0.23$ & $5.70 \pm 0.24$
\end{tabular}

${ }^{a_{T}}$ The $\mathrm{p} K_{\mathrm{a} 1}$ value arises from the cysteine nucleophile and $\mathrm{p} K_{\mathrm{a} 2}$ from the aspartic acid.

versus 6 , and in the variant, it is reduced to 4.8 . The variant and native $\mathrm{YopH}$ have very similar activities at their optimal $\mathrm{pH}$, but the variant has significantly more activity at $\mathrm{pH}<5$, by approximately an order of magnitude at $\mathrm{pH} 4.5$.

The fits of $\mathrm{pH}$-rate data to eq 1 show negligible changes to the $\mathrm{p} K_{\mathrm{a}}$ of the aspartic acid, reflected in the basic limb in the variants, but clear changes are present in the acidic limb, as reflected in the kinetic $\mathrm{p} K_{\mathrm{a} 1}$ values in Table 2 . The $\mathrm{T} 177 \mathrm{G}$ mutation in PTP1B raises the kinetic $\mathrm{p} K_{\mathrm{a} 1}$ by 0.36 units, whereas the orthogonal G352T mutation in YopH results in a full unit decrease.

The thermodynamic $\mathrm{p} K_{\mathrm{a}}$ of the Cys nucleophile depends on the stabilization of the thiolate form provided by the neighboring histidine residue. Although no significant perturbation to this interaction was seen in our X-ray structures, or arose in the simulations, unobserved structural effects could be significant. For example, local protein unfolding in staphylococcal nuclease was shown to alter $\mathrm{p} K_{\mathrm{a}}$ values and, conversely, affect the $\mathrm{pH}$ dependence of local protein fluctuations. ${ }^{50}$ It was important to determine whether unseen long-range structural effects of the mutation might affect the thermodynamic $\mathrm{p} K_{\mathrm{a}}$ of the active-site Cys residue. This value was measured using the $\mathrm{pH}$ dependency of the rate of inactivation of the enzymes by iodoacetate, a method that has been applied previously in a number of proteins, including the PTPs YopH and VHR. ${ }^{7,35}$ The thermodynamic $\mathrm{p} K_{\mathrm{a}}$ of the native YopH has been reported as $4.67 \pm 0.15$. $^{7^{\circ}}$ Using conditions described in the Materials and Methods, we obtained the $\mathrm{pH}$ dependency for inactivation plotted in Figure S4, which yielded a value for the G352T of $4.3 \pm 0.2$ and $4.6 \pm$ 0.2 for native YopH. Thus, the different $\mathrm{pH}$ dependency of the G352T variant arises not from substantial alteration of the thermodynamic $\mathrm{p} K_{\mathrm{a}}$ of the thiol group of the cysteine side chain but predominantly from altered dynamic properties that affect the factors described in the Introduction, which distort the kinetic $\mathrm{p} K_{\mathrm{a}}$ values from their thermodynamic values and affect the $\mathrm{pH}$ dependency of catalysis.

The crystal structures showing both ligand-free mutants crystallize in the loop-closed conformation suggest these mutations result in a shifted equilibrium to favor their closed-loop conformations. The crystal structures do not reveal obvious changes from the mutations that would rationalize a shift in equilibrium between the open and closed loops. Computational modeling was therefore used to assess how these mutations affect the respective populations of WPDloop-open and -closed conformations.

\section{Computational Investigations into the Origin of the Implied Population Shifts}

Our structural and kinetic analysis presented above demonstrate that the point mutations T177G in PTP1B and YopH G352T can alter the relative populations of the closed and open states of the WPD-loop. In order to help unravel the molecular mechanism(s) behind these population shifts, we performed MD simulations on both WT PTP1B and WT YopH and their respective point variants (T177G PTP1B and G352T YopH). For all four systems, we performed 10 replicas of $100 \mathrm{~ns}$ long MD simulations starting from the both the closed and open states ( $2 \mu$ s per system in total). The use of multiple replicas (like the 10 performed here) is important for obtaining both reliable and reproducible MD simulation data. ${ }^{51}$ We note that both we ${ }^{18,52}$ and others ${ }^{53,54}$ performed previous detailed computational studies of loop dynamics (or other large-scale conformational changes) using advanced enhanced sampling approaches that are able to (at least to some extent) quantitatively evaluate the populations observed between the different conformational states. This includes recent computational studies of WPD-loop dynamics in wildtype PTP1B and YopH. ${ }^{18}$ However, such studies are far from computationally trivial. In addition, while relevant NMR data exist that quantify the population difference between the two states in the wild-type enzymes, ${ }^{17}$ these differences are both quite subtle (in the range of $1-2 \mathrm{kcal} \mathrm{mol}^{-1}$ depending on whether a ligand is bound or not, making them hard to quantify computationally), and there also do not exist analogous data for the substituted variants studied in this work. However, as discussed below, our conventional MD simulations appear to be able to capture the population shift between different conformational states upon amino acid substitution and key qualitative features associated with this shift.

To assess the overall stability of the closed and open WPDloop conformations for each system, we measured the $\mathrm{C}_{\alpha}$ RMSD of the WPD-loop over the course of each simulation relative to its starting structure (i.e., X-ray structure with a closed or open WPD-loop). Focusing first on the closed state simulations (Figure 6A), we found that both point variants have a higher occupancy of sampling low RMSD states (i.e., those representing a closed WPD-loop) compared to that of their counterpart WT PTPs. These results are consistent with 
A

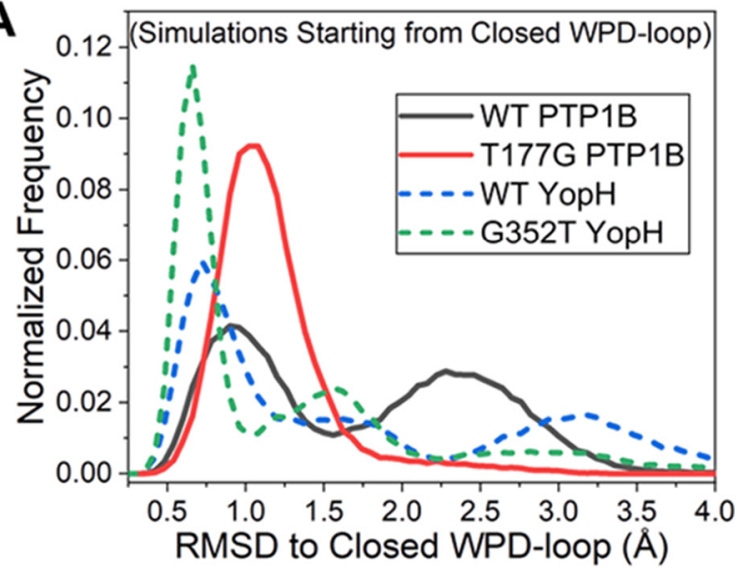

B

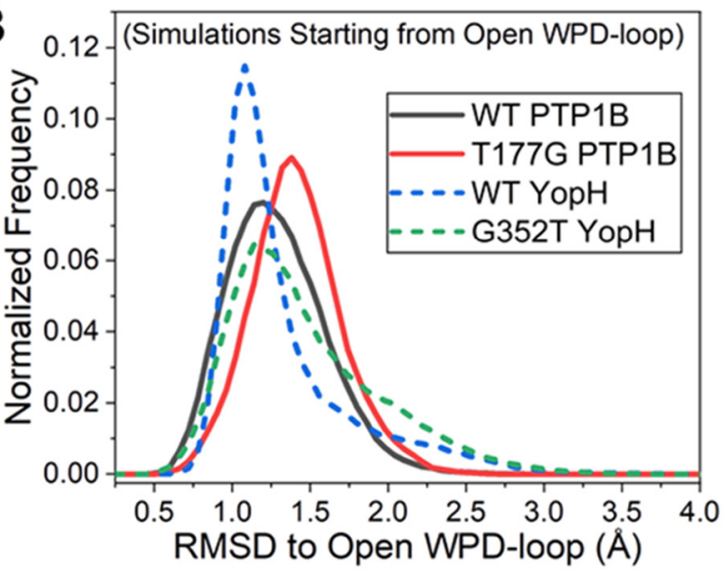

C

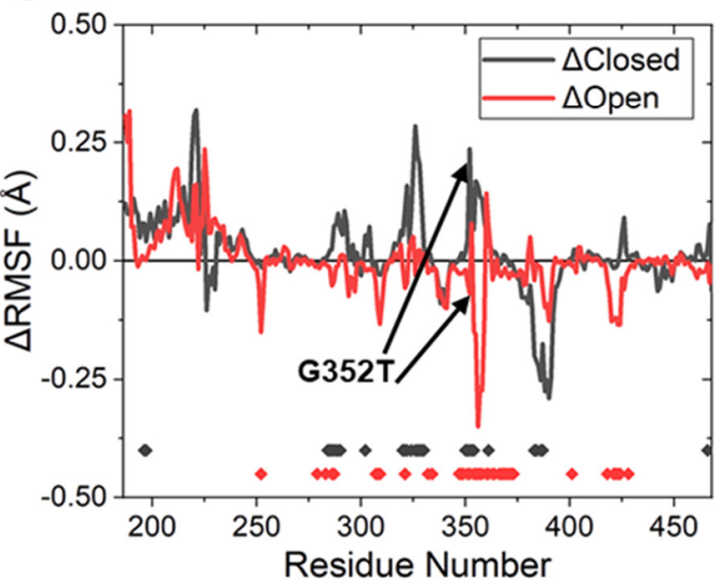

E

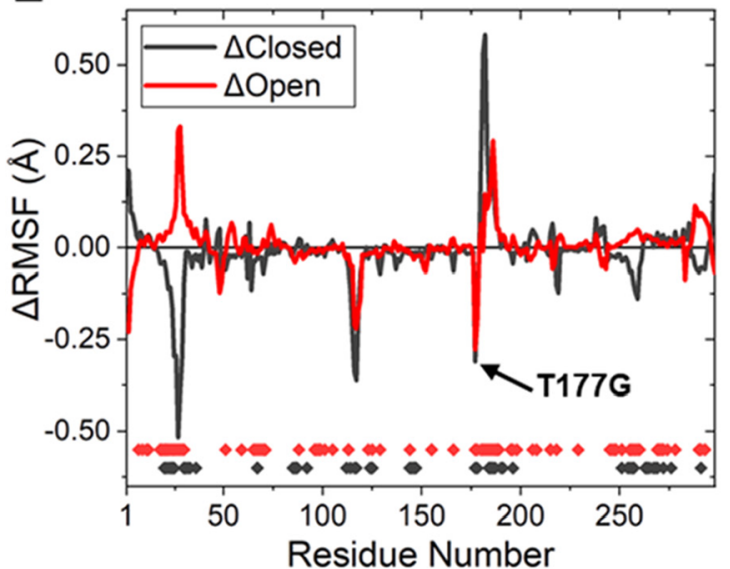

D $\Delta$ Closed WT-G352T

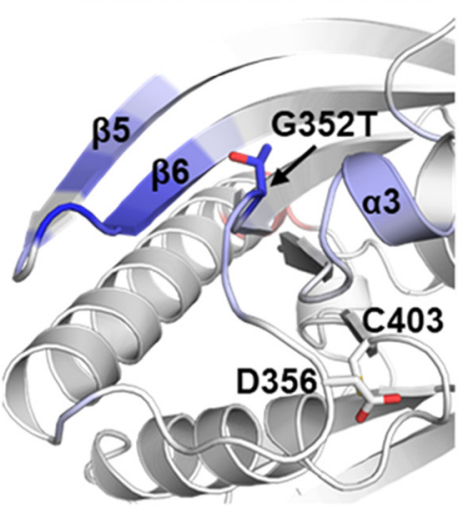

F $\Delta$ Closed WT-T177G

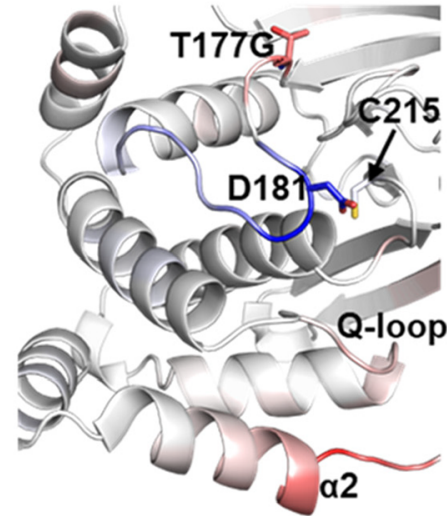

$\Delta$ Open WT-G352T

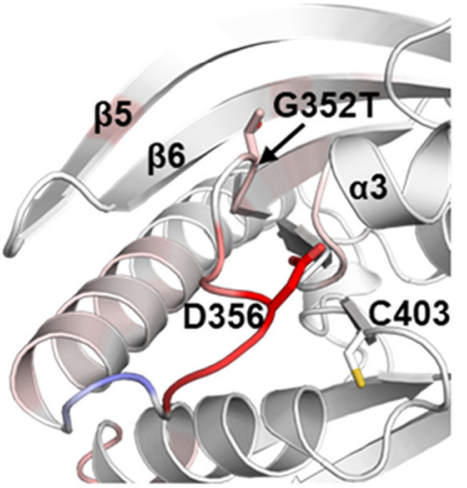

$\Delta$ Open WT-T177G

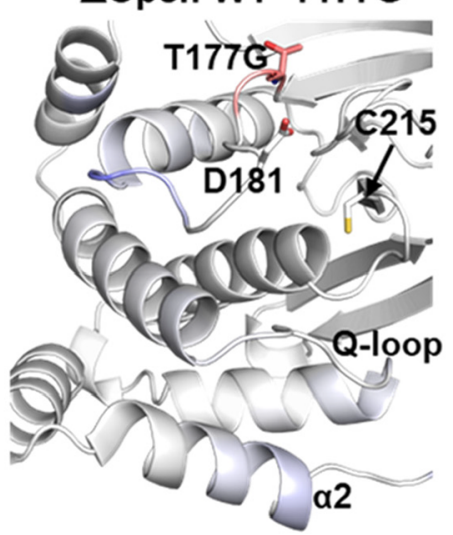

Figure 6. $(\mathrm{A}, \mathrm{B})$ Histograms of the $\mathrm{C}_{\alpha}$ RMSD of the WPD-loop relative to its starting (crystal structure) position, obtained from $10 \times 100 \mathrm{~ns}$ MD simulations starting from either the (A) closed or (B) open WPD-loop conformations. (C) Differences in calculated per residue $\mathrm{C}_{\alpha}$ root mean square fluctuation ( $\triangle \mathrm{RMSF}$ ) for the closed and open states between the WT and G352T variant of YopH, with a more positive value indicating increased flexibility in the WT residue. All simulations were performed on the ligand-free form of the enzyme. The small dots at the bottom of the graph represent $\Delta$ RMSF values that are identified as statistically significant as determined by a two-sample $t$-test $(p<0.05)$. Significant $\Delta$ RMSF differences for closed and open states are marked as black and red dots, respectively. (D) Color mapping of the significantly different (as determined by the above-described $t$-test) $\Delta$ RMSF values onto the structure of YopH. Color mapping is performed from blue (positive $\Delta$ RMSF) through to white ( $0 \Delta$ RMSF value or not determined to be significantly different) to red (negative $\Delta$ RMSF). In practice, a blue residue would mean increased rigidity for the G352T variant over the WT, whereas a red residue would indicate increased flexibility of the G352T variant. (E,F) Same set of data as described for YopH in panels C and D but now for PTP1B instead (and the mutation T177G instead of G352T).

our X-ray data, in which, for both РТР1B and YopH, the ligand-free form is observed with a closed WPD-loop conformation (Figure 3). Interestingly, we also observe a (subtle) reduction in the stability of the open WPD-loop conformation for both point variants (Figure 6B), as their histograms are broader and generally sample greater RMSD values than their counterpart WT PTPs. This would therefore suggest that stabilization of both the closed state and 
A

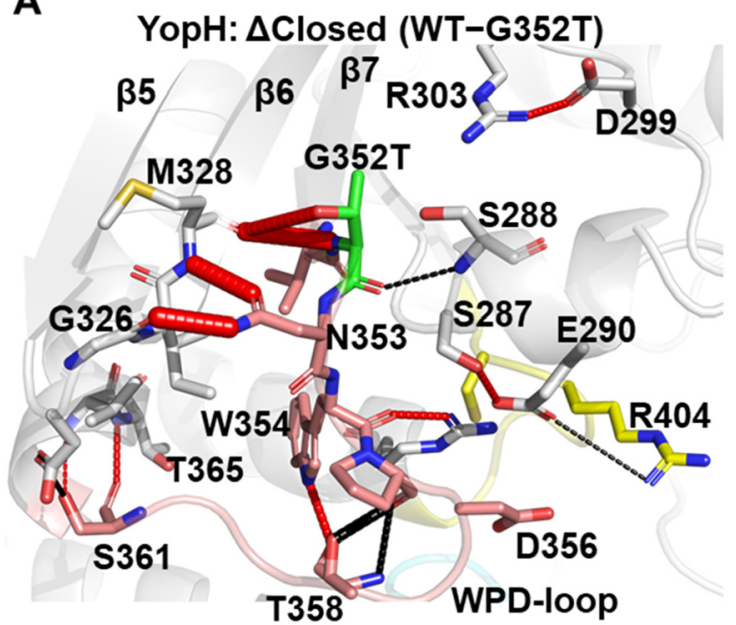

C

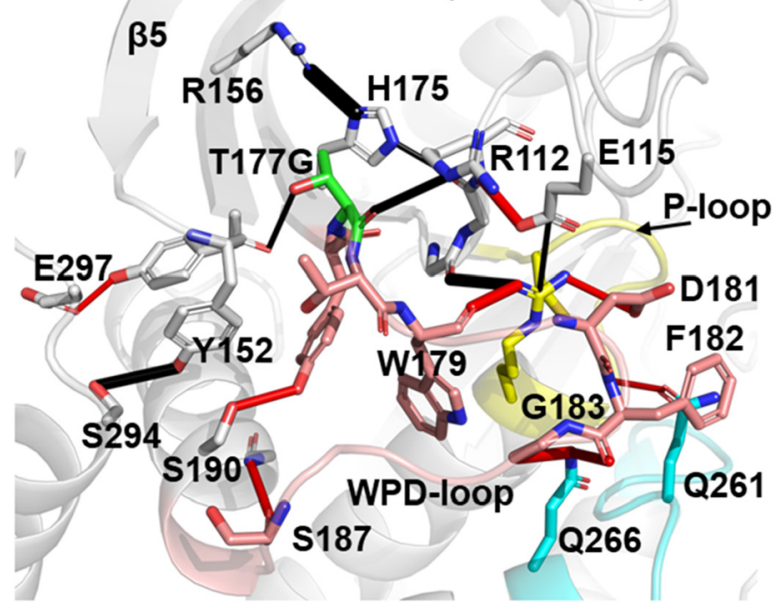

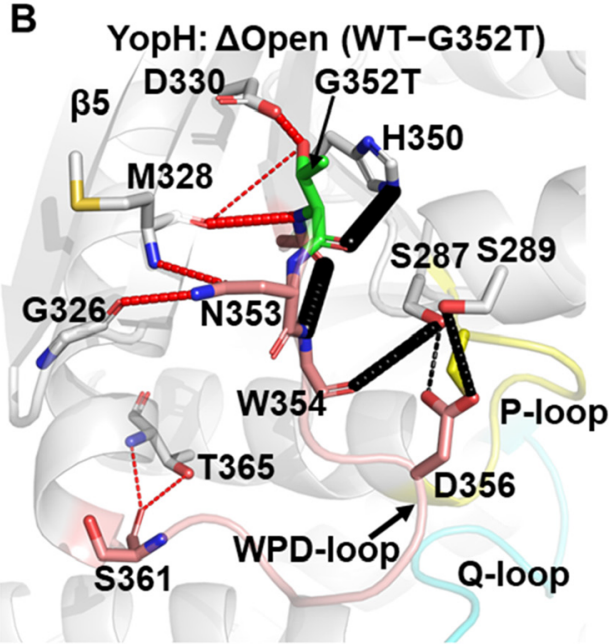

D

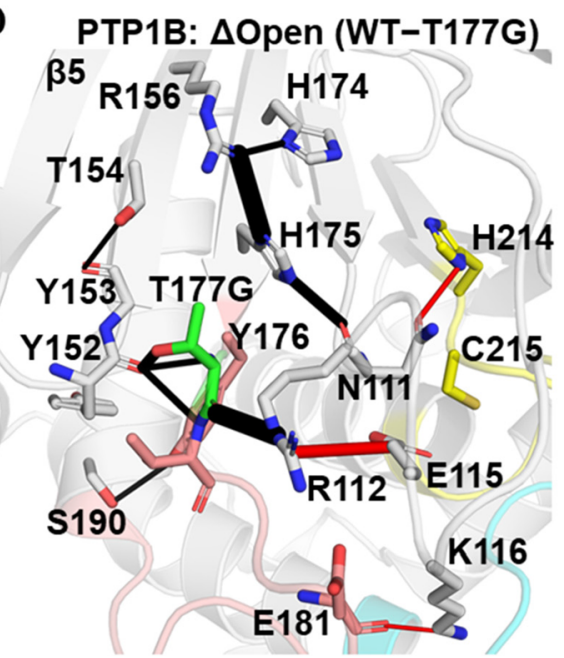

Figure 7. (A,B) Differences between the hydrogen bonding (H-bonding) interaction networks formed for the WT and G352T point variant of YopH in the WPD-loop (A) closed and (B) open conformations. H-bonding interactions which have a higher occupancy in the WT are shown as black dashes between the donor and acceptor atom, with red dashes used to indicate H-bonding interactions which have a higher occupancy in the G352T variant. The width of the dash indicates the magnitude of the difference in the occupancy of the hydrogen bond between the two enzymes. (C,D) These panels show the same data as shown for YopH in panels A and B but now illustrate instead the differences between WT and T177G PTP1B in their WPD-loop (C) closed and (D) open WPD-loop states, respectively.

destabilization of the open state are responsible for driving the experimentally observed population shifts toward the closed state for both point variants.

Our RMSD data presented above reflect differences in the overall stability of the WPD-loop, but in order to localize this effect to the per-residue level, we determined how the flexibility of the enzymes differed for both the closed and open states of the WPD-loop for the WT PTP against its counterpart point variant (Figure $6 \mathrm{C}-\mathrm{F}$ ). Given that our RMSD analysis above showed significant sampling of WPDloop conformational states different from the starting structures, we only took forward MD simulation snapshots within $2 \AA$ of the reference (crystal structure WPD-loop closed or open) state for analysis. Further, in order to ensure the differences identified were statistically significant, we performed a two-sample $t$-test ( $p<0.05$ used as cutoff) using the 10 replicas performed per system.

Focusing first on the differences in flexibility between the closed states of WT and G352T YopH (Figure 6C,D), we identified the G352T substitution to significantly rigidify the
$\mathrm{N}$-terminal portion of the WPD-loop where the substitution is located, alongside the nearby $\beta 5$ and $\beta 6$ strands and the $\alpha 3$ helix. For the open WPD-loop conformation, our MD simulations (Figure 6C,D) show that the G352T mutation has increased the flexibility of both the central and C-terminal portions of the WPD-loop alongside the $\alpha 4$-helix (which is directly connected to the C-terminal portion of the WPDloop). These results are therefore consistent with our RMSD data (Figure 6A,B), in which both the closed state stabilization (including nearby secondary structure elements) and open state destabilization are responsible for the experimentally observed population shift toward the closed state for G352T YopH.

For the differences in flexibility between WT PTP1B and its T177G point variant (Figure 6E,F), we observe the point variant to be directly destabilizing toward both its own position on the loop and that of the neighboring T188 residue in both the open and closed states of the WPD-loop. Given the nature of the mutation (threonine to the less conformationally constrained glycine) and resulting loss of side chain 
interactions, these results are unsurprising. Interestingly, however, this mutation has an indirect (and compensatory) effect of rigidifying parts of the central and C-terminal portions for both the WPD-loop's closed and open states, with the magnitude of this rigidification being notably stronger for the closed state of the WPD-loop (Figure 6E,F). Taken together with our RMSD analysis (Figure 6A,B), this compensatory effect appears to result in an overall more rigid WPD-loop for T177G PTP1B when in the closed state, in contrast to a more flexible WPD-loop open state for T177G PTP1B (when compared to WT PTP1B).

Interestingly, we also observe the $\mathrm{T} 177 \mathrm{G}$ mutation to alter the stability of residues around the Q-loop and $\alpha 2$ - and $\alpha 5$ helices. These regions have recently been implicated to be part of the PTP1B allosteric network through a combined mutagenesis and bioinformatics study. ${ }^{55}$ The sensitivity of this region to the $\mathrm{T} 177 \mathrm{G}$ point mutation observed in our MD simulations for both the closed and open states adds further weight to this argument.

Changes in the Local Hydrogen Bonding Network Help Rationalize the Observed Population Shifts

Our RMSD and RMSF calculations described above helped identify how both point variants altered the stability of both the open and closed states of the WPD-loop relative to their counterpart WT PTPs. To understand how these structural effects manifest, we analyzed the changes in the hydrogen bonding (H-bonding) interaction networks between both WT PTPs and their respective point variants for both the closed and open states (Figure 7). We note that while we utilized multiple replica simulations to reduce the risk of noise based differences in H-bonding interactions, we further limited our search to differences in $\mathrm{H}$-bonding interactions close to the mutation site or on the WPD-loop itself and, further, only included those interactions with differences of at least $15 \%$ greater occupancy in one state over the other (see the Materials and Methods for further details).

For YopH and the G352T variant in the WPD-loop closed state (Figure 7A), we observe the G352T variant to form higher occupancy interactions with the backbone carbonyl of M328 on the $\beta 6$-strand (both through the main chain nitrogen and the side chain hydroxyl group only present in the point variant). Further, the neighboring N353 side chain forms notably higher occupancy $\mathrm{H}$-bonding interactions with the backbones of M328 and G326 than in the WT simulations. These higher occupancy interactions help explain the increased rigidity observed in the loop closed state for both the WPDloop and surrounding regions (Figure 6C,D).

For YopH in the open state (Figure 7B), while many of the same interactions as described for the closed state are stronger (but to a lesser extent) in the point variant, we also observe the WT to make many higher occupancy hydrogen bonds. For example, G352 makes significantly higher occupancy interactions with $\mathrm{H} 350$ and W354, which would suggest the additional conformational restriction placed on the backbone dihedrals (in the point variant G352T) prevents/reduces the sampling of these stabilizing interactions in the open state.

Focusing for now on the differences between WT PTP1B in both the open and closed WPD-loop states, the direct impact of the T177G mutation is clearly detrimental toward its own interactions with Y152 and R112, with reduced occupancy of hydrogen bonding interactions compared to what is observed in the WT enzyme (Figure $7 \mathrm{C}, \mathrm{D}$ ). The T177A substitution was previously identified by Cui et al. ${ }^{49}$ to also induce a population shift through destabilization of the open state (by disrupting an interaction network between T178, T177, and H175). Here, we show an extension of the disruption of this network following from $\mathrm{H} 175$ to R156 of the $\beta 6$-strand and $\mathrm{N} 111$ and E112 on the E-loop (Figure 7C,D). We note that while this same network is disturbed in both the open and closed states upon mutation (which is consistent with the immediate site surrounding the WPD-loop becoming more unstable as seen in our RMSF calculations, Figure $6 \mathrm{E}, \mathrm{F}$ ), the effect appears to be stronger in the open state, especially when considering R112's H-bonding interaction with the backbone of T177G. Further, for the closed state of PTP1B, we observed differences in the hydrogen bonding patterns of the closed WPD-loop conformation between residues on the central portion of the WPD-loop (W179, D181, F182, G183) and the neighboring Q- and P-loops (Figure $7 \mathrm{C}$ ), likely explaining the increased rigidity for this region seen in our RMSF calculations (Figure 6E,F).

\section{CONCLUSIONS}

Catalysis in PTPs relies on the closure of a flexible protein loop carrying a residue acting as a general acid in the first step of the mechanism and as a base in the second step. The orthogonal substitutions of a single, noncatalytic residue in the WPD-loops of YopH and PTP1B result in no loss of maximal catalytic activity but in shifted $\mathrm{pH}$-rate profiles exhibiting an altered kinetic $\mathrm{p} K_{\mathrm{a}}$ of the nucleophilic cysteine residue. Surprisingly, the G352T variant of YopH has no loss of activity at optimal $\mathrm{pH}$ but activity at low $\mathrm{pH}$ significantly higher than that of native $\mathrm{YopH}$, reflected in a broadened $\mathrm{pH}$-rate profile on the acid side. Fitting the $\mathrm{pH}$-rate data reveals a significantly lower apparent, or kinetic, $\mathrm{p} K_{\mathrm{a}}$ of the nucleophilic C402. Titration of this residue showed a very modest change in the thermodynamic $\mathrm{p} K_{\mathrm{a}}$, meaning the altered kinetic $\mathrm{p} K_{\mathrm{a}}$ results from changes in other processes affecting the catalytic mechanism. Changes in the corresponding PTP1B T177G variant are more modest and in the opposite direction; the $\mathrm{pH}$ profile is slightly narrowed, and the variant is less active in the most acidic range of the profile. Crystal structures show no structural perturbations result from the substitutions but imply the WPD-loop closed conformation has become more favored in the ligand-free enzyme, in contrast to the native enzyme.

Our computational analysis further supports the conclusion that both variants have a higher occupancy of the loop-closed conformation than their WT counterparts and shows that the shift results from a combination of increased stability of the closed state of the WPD-loop, with concomitant destabilization of the corresponding loop-open state. Simulations identified the origins of this population shift and also revealed unexpected differences in the flexibility of the WPD-loop and neighboring regions that result from these mutations, showing these single point mutations can substantially disrupt the conformational dynamics of many residues in the active site and neighboring regions. Specifically, relative to the native enzyme, the G352T substitution in YopH makes the $\mathrm{N}$ terminal portion of its WPD-loop and nearby secondary structural elements more rigid in the loop-closed state, while simultaneously increasing the flexibility of the central and Cterminal portions of the loop in the open state. In the case of PTP1B, the T177G substitution results in an obvious loss of hydrogen bonding interactions at the site of substitution in both the closed and open states. However, when combined 
with changes to the remainder of the WPD-loop, it results in a more rigid closed loop and a more flexible open loop.

The involvement of nonchemical processes in perturbing the observed $\mathrm{p} K_{\mathrm{a}}$ values of ionizable catalytic residues obtained from enzyme kinetics studies has been well-documented. ${ }^{21-23}$

While this can be a bane to enzymologists, it affords nature a tool to evolve the $\mathrm{pH}$ dependency of an enzyme by a more facile and less disruptive manner than altering the electrostatic network around catalytic residues themselves or changing them. Protein motions are one such process that, as the results shown here demonstrate, can be altered in a manner such that a significant shift in the $\mathrm{pH}$ dependence of activity can result. Although an area of growing study, the role of protein dynamics in phosphatases is less well-known than in their catalytic counterparts, kinases, where significant roles of protein motions are well-documented. ${ }^{56,57}$ Aside from three highly conserved residues, PTPs have significant sequence variation within their WPD-loops (Figure S1), the mobility of which is critical for catalysis. Those differences, and the altered dynamics of loop motion that result, likely contribute to the variation in $\mathrm{pH}$ dependency seen among the PTP family despite their shared catalytic residues and mechanism. The results presented here mean that evaluating the effect of mutations in PTPs, or other enzymes, by comparing the activity of variants with the WT enzyme at a single $\mathrm{pH}$ may not give a complete picture of the actual impact of the amino acid substitutions. More generally, these results show a previously unappreciated means by which nature can alter the $\mathrm{pH}$ dependency of catalysis, by employing a phenomenon that bedevils the interpretation of $\mathrm{pH}$-rate profiles by experimentalists.

\section{ASSOCIATED CONTENT}

\section{Supporting Information}

The Supporting Information is available free of charge at https://pubs.acs.org/doi/10.1021/jacsau.1c00054.

Additional simulation methodology details and supplemental structural analysis (PDF)

\section{AUTHOR INFORMATION}

\section{Corresponding Authors}

Sean J. Johnson - Department of Chemistry and Biochemistry, Utah State University, Logan, Utah 84322-0300, United

States; 10 orcid.org/0000-0001-7992-2494;

Email: sean.johnson@usu.edu

Alvan C. Hengge - Department of Chemistry and Biochemistry, Utah State University, Logan, Utah 843220300, United States; (1) orcid.org/0000-0002-5696-2087; Email: alvan.hengge@usu.edu

Shina C. L. Kamerlin - Science for Life Laboratory, Department of Chemistry - BMC, Uppsala University, $S$ 75123 Uppsala, Sweden; (1) orcid.org/0000-0002-31901173; Email: lynn.kamerlin@kemi.uu.se

\section{Authors}

Ruidan Shen - Department of Chemistry and Biochemistry, Utah State University, Logan, Utah 84322-0300, United States

Rory M. Crean - Science for Life Laboratory, Department of Chemistry - BMC, Uppsala University, S-751 23 Uppsala, Sweden
Complete contact information is available at: https://pubs.acs.org/10.1021/jacsau.1c00054

Notes

The authors declare no competing financial interest.

\section{ACKNOWLEDGMENTS}

This work was supported by the Carl Tryggers Foundation for Scientific Research (postdoctoral fellowship to R.M.C., Grant No. CTS 19:172), the Knut and Alice Wallenberg Foundation (Wallenberg Academy Fellowship to S.C.L.K., Grant No. 2018.0140), the Human Frontier Science Program (Grant No. RGP0041/2017), and the Swedish Research Council (Grant No. 2019-03499). Computational resources were provided by the Swedish National Infrastructure for Computing (Grant Nos. 2019/2-1, 2019/3-258, and 2020/5-250), and simulations were performed on the Tetralith Cluster at NSC, Linköping. Funding for the in-house X-ray generator was provided by NSF-MRI Award DBI1228874. Use of the Stanford Synchrotron Radiation Lightsource, SLAC National Accelerator Laboratory, is supported by the U.S. Department of Energy, Office of Science, Office of Basic Energy Sciences under Contract No. DE-AC02-76SF00515. The SSRL Structural Molecular Biology Program is supported by the DOE Office of Biological and Environmental Research and by the National Institutes of Health, National Institute of General Medical Sciences (P30GM133894). The contents of this publication are solely the responsibility of the authors and do not necessarily represent the official views of NIGMS or NIH. Finally, we thank Darko Mitrovic (KTH) for his assistance with the bioinformatic analysis.

\section{REFERENCES}

(1) Yun, H. Y.; Lee, J.; Kim, H.; Ryu, H.; Shin, H. C.; Oh, B. H.; Ku, B.; Kim, S. J. Structural Study Reveals the Temperature-Dependent Conformational Flexibility of Tk-PTP, a Protein Tyrosine Phosphatase from Thermococcus Kodakaraensis KOD1. PLoS One 2018, 13, No. e0197635.

(2) LaForgia, S.; Morse, B.; Levy, J.; Barnea, G.; Cannizzaro, L. A.; Li, F.; Nowell, P. C.; Boghosian-Sell, L.; Glick, J.; Weston, A.; et al. Receptor Protein-Tyrosine Phosphatase Gamma is a Candidate Tumor Suppressor Gene at Human Chromosome Region 3p21. Proc. Natl. Acad. Sci. U. S. A. 1991, 88, 5036-5040.

(3) Denu, J. M.; Dixon, J. E. Protein Tyrosine Phosphatases: Mechanisms of Catalysis and Regulation. Curr. Opin. Chem. Biol. 1998, 2, 633-641.

(4) Jackson, M. D.; Denu, J. M. Molecular Reactions of Protein Phosphatases-Insights From Structure and Chemistry. Chem. Rev. 2001, 101, 2313-2340.

(5) Zhang, Z. Y. Protein Tyrosine Phosphatases: Structure and Function, Substrate Specificity, and Inhibitor Development. Annu. Rev. Pharmacol. Toxicol. 2002, 42, 209-234.

(6) Zhang, Z. Y. Mechanistic Studies on Protein Tyrosine Phosphatases. Prog. Nucleic Acid Res. Mol. Biol. 2003, 73, 171-220.

(7) Zhang, Z. Y.; Dixon, J. E. Active Site Labeling of the Yersinia Protein Tyrosine Phosphatase: the Determination of the $\mathrm{p} K_{\mathrm{a}}$ of the Active Site Cysteine and the Function of the Conserved Histidine 402. Biochemistry 1993, 32, 9340-9345.

(8) Zhang, Z. Y.; Palfey, B. A.; Wu, L.; Zhao, Y. Catalytic Function of the Conserved Hydroxyl Group in the Protein Tyrosine Phosphatase Signature Motif. Biochemistry 1995, 34, 16389-16396.

(9) Zhao, Y.; Zhang, Z. Y. Reactivity of Alcohols Toward the Phosphoenzyme Intermediate in the Protein-Tyrosine PhosphataseCatalyzed Reaction: Probing the Transition State of the Dephosphorylation Step. Biochemistry 1996, 35, 11797-11804. 
(10) Brandao, T. A.; Hengge, A. C.; Johnson, S. J. Insights into the Reaction of Protein-Tyrosine Phosphatase 1B: Crystal Structures for Transition State Analogs of Both Catalytic Steps. J. Biol. Chem. 2010, $285,15874-15883$.

(11) Brandao, T. A.; Johnson, S. J.; Hengge, A. C. The Molecular Details of WPD-Loop Movement Differ in the Protein-Tyrosine Phosphatases YopH and PTP1B. Arch. Biochem. Biophys. 2012, 525, $53-59$.

(12) Fauman, E. B.; Yuvaniyama, C.; Schubert, H. L.; Stuckey, J. A.; Saper, M. A. The X-ray Crystal Structures of Yersinia Tyrosine Phosphatase with Bound Tungstate and Nitrate. Mechanistic Implications. J. Biol. Chem. 1996, 271, 18780-18788.

(13) Groves, M. R.; Yao, Z. J.; Roller, P. P.; Burke, T. R., Jr.; Barford, D. Structural Basis for Inhibition of the Protein Tyrosine Phosphatase 1B by Phosphotyrosine Peptide Mimetics. Biochemistry 1998, 37, 17773-17783.

(14) Stuckey, J. A.; Schubert, H. L.; Fauman, E. B.; Zhang, Z. Y.; Dixon, J. E.; Saper, M. A. Crystal Structure of Yersinia Protein Tyrosine Phosphatase at $2.5 \mathrm{~A}$ and the Complex with Tungstate. Nature 1994, 370, 571-575.

(15) Ala, P. J.; Gonneville, L.; Hillman, M. C.; Becker-Pasha, M.; Wei, M.; Reid, B. G.; Klabe, R.; Yue, E. W.; Wayland, B.; Douty, B.; Polam, P.; Wasserman, Z.; Bower, M.; Combs, A. P.; Burn, T. C.; Hollis, G. F.; Wynn, R. Structural Basis for Inhibition of ProteinTyrosine Phosphatase 1B by Isothiazolidinone Heterocyclic Phosphonate Mimetics. J. Biol. Chem. 2006, 281, 32784-32795.

(16) Barford, D.; Flint, A. J.; Tonks, N. K. Crystal Structure of Human Protein Tyrosine Phosphatase 1B. Science 1994, 263, 13971404.

(17) Whittier, S. K.; Hengge, A. C.; Loria, J. P. Conformational Motions Regulate Phosphoryl Transfer in Related Protein Tyrosine Phosphatases. Science 2013, 341, 899-903.

(18) Crean, R. M.; Biler, M.; van der Kamp, M. W.; Hengge, A. C.; Kamerlin, S. C. L. Loop Dynamics and Enzyme Catalysis in Protein Tyrosine Phosphatases. J. Am. Chem. Soc. 2021, 143, 3830-3845.

(19) Tonks, N. K.; Diltz, C. D.; Fischer, E. H. Characterization of the Major Protein-Tyrosine-Phosphatases of Human Placenta. J. Biol. Chem. 1988, 263, 6731-6737.

(20) Zhang, Z.Y.; Malachowski, W.P.; Van Etten, R.L.; Dixon, J.E. Nature of the Rate-Determining Steps of the Reaction Catalyzed by the Yersinia Protein-Tyrosine Phosphatase. J. Biol. Chem. 1994, 269, $8140-8145$.

(21) Alberty, R. A.; Massey, V. On the Interpretation of the $\mathrm{pH}$ Variation of the Maximum Initial Velocity of an Enzyme-Catalyzed Reaction. Biochim. Biophys. Acta 1954, 13, 347-353.

(22) Alberty, R. A.; Peller, L. Multiple Intermediates in Steady State Enzyme Kinetics. I. The Mechanism Involving a Single Substrate and Product. J. Am. Chem. Soc. 1959, 81, 5907-5914.

(23) Knowles, J. R.; Jencks, W. P. The Intrinsic $p K_{a}$ Values of Functional Groups in Enzymes: Improper Deductions from the $\mathrm{pH}$ Dependence of Steady-State Parameters. Crit. Rev. Biochem. 1976, 4, $165-173$.

(24) Cook, P. F.; Cleland, W. W. Enzyme Kinetics and Mechanism; Taylor \& Francis Group, LLC, 2007.

(25) Fierke, C. A.; Johnson, K. A.; Benkovic, S. J. Construction and Evaluation of the Kinetic Scheme Associated with Dihydrofolate Reductase from Escherichia Coli. Biochemistry 1987, 26, 4085-4092.

(26) Harris, T. K.; Turner, G. J. Structural Basis of Perturbed $\mathrm{p} K_{\mathrm{a}}$ Values of Catalytic Groups in Enzyme Active Sites. IUBMB Life 2002, $53,85-98$.

(27) Hengge, A. C.; Sowa, G. A.; Wu, L.; Zhang, Z. Y. Nature of the Transition State of the Protein-Tyrosine Phosphatase-Catalyzed Reaction. Biochemistry 1995, 34, 13982-13987.

(28) Hollfelder, F.; Herschlag, D. The Nature of the Transition State for Enzyme-Catalyzed Phosphoryl Transfer. Hydrolysis of O-aryl Phosphorothioates by Alkaline Phosphatase. Biochemistry 1995, 34, $12255-12264$.

(29) Zhang, Z. Y.; Clemens, J. C.; Schubert, H. L.; Stuckey, J. A.; Fischer, M. W.; Hume, D. M.; Saper, M. A.; Dixon, J. E. Expression,
Purification, and Physicochemical Characterization of a Recombinant Yersinia Protein Tyrosine Phosphatase. J. Biol. Chem. 1992, 267, 23759-23766.

(30) Afonine, P. V.; Grosse-Kunstleve, R. W.; Echols, N.; Headd, J. J.; Moriarty, N. W.; Mustyakimov, M.; Terwilliger, T. C.; Urzhumtsev, A.; Zwart, P. H.; Adams, P. D. Towards Automated Crystallographic Structure Refinement with Phenix.refine. Acta Crystallogr., Sect. D: Biol. Crystallogr. 2012, 68, 352-367.

(31) Liebschner, D.; Afonine, P. V.; Baker, M. L.; Bunkoczi, G.; Chen, V. B.; Croll, T. I.; Hintze, B.; Hung, L. W.; Jain, S.; McCoy, A. J.; Moriarty, N. W.; Oeffner, R. D.; Poon, B. K.; Prisant, M. G.; Read, R. J.; Richardson, J. S.; Richardson, D. C.; Sammito, M. D.; Sobolev, O. V.; Stockwell, D. H.; Terwilliger, T. C.; Urzhumtsev, A. G.; Videau, L. L.; Williams, C. J.; Adams, P. D. Macromolecular Structure Determination Using X-rays, Neutrons and Electrons: Recent Developments in Phenix. Acta Crystallogr., Sect. D: Biol. Crystallogr. 2019, 75, 861-877.

(32) Moise, G.; Gallup, N. M.; Alexandrova, A. N.; Hengge, A. C.; Johnson, S. J. Conservative Tryptophan Mutants of the Protein Tyrosine Phosphatase YopH Exhibit Impaired WPD-loop Function and Crystallize with Divanadate Esters in their Active Sites. Biochemistry 2015, 54, 6490-6500.

(33) Emsley, P.; Lohkamp, B.; Scott, W. G.; Cowtan, K. Features and Development of Coot. Acta Crystallogr., Sect. D: Biol. Crystallogr. 2010, 66, 486-501.

(34) Cleland, W. W. Determining the Chemical Mechanisms of Enzyme-Catalyzed Reactions by Kinetic Studies. In Advances in Enzymology 2006, 45, 273-387.

(35) Denu, J. M.; Zhou, G.; Guo, Y.; Dixon, J. E. The Catalytic Role of Aspartic acid-92 in a Human Dual-Specific Protein- TyrosinePhosphatase. Biochemistry 1995, 34, 3396-3403.

(36) Case, D. A.; Ben-Shalom, I. Y.; Brozell, S. R.; Cerutti, D. S.; Cheatham, T. E., III; Cruzeiro, V. W. D.; Darden, T. A.; Duke, R. E.; Ghoreishi, D.; Gilson, M. K.; Gohlke, H.; Goetz, A. W.; Greene, D.; Harris, R.; Homeyer, N.; Izadi, S.; Kovalenko, A.; Kurtzman, T.; Lee, T. S.; LeGrand, S.; Li, P.; Lin, C.; Liu, J.; Luchko, T.; Luo, R.; Mermelstein, D. J.; Merz, K. M.; Miao, Y.; Monard, G.; Nguyen, C.; Nguyen, H.; Omelyan, I.; Onufriev, A.; Pan, F.; Qi, R.; Roe, D. R.; Roitberg, A.; Sagui, C.; Schott-Verdugo, S.; Shen, J.; Simmerling, C. L.; Smith, J.; Salomon-Ferrer, R.; Swails, J.; Walker, R. C.; Wang, J.; Wei, H.; Wolf, R. M.; Wu, X.; Xiao, L.; York, D. M.; Kollman, P. A. AMBER 2018; University of California: San Francisco, CA, 2018.

(37) Maier, J. A.; Martinez, C.; Kasavajhala, K.; Wickstrom, L.; Hauser, K. E.; Simmerling, C. ff14SB: Improving the Accuracy of Protein Side Chain and Backbone Parameters from ff99SB. J. Chem. Theory Comput. 2015, 11, 3696-3713.

(38) Jorgensen, W. L.; Chandrasekhar, J.; Madura, J. D.; Impey, R. W.; Klein, M. L. Comparison of Simple Potential Functions for Simulating Liquid Water. J. Chem. Phys. 1983, 79, 926-935.

(39) Keedy, D. A.; Hill, Z. B.; Biel, J. T.; Kang, E.; Rettenmaier, T. J.; Brandão-Neto, J.; Pearce, N. M.; von Delft, F.; Wells, J. A.; Fraser, J. S. An Expanded Allosteric Network in PTP1B by Multitemperature Crystallography, Fragment Screening, and Covalent Tethering. eLife 2018, 7, e36307.

(40) Denu, J. M.; Lohse, D. L.; Vijayalakshmi, J.; Saper, M. A.; Dixon, J. E. Visualization of Intermediate and Transition-State Structures in Protein-Tyrosine Phosphatase Catalysis. Proc. Natl. Acad. Sci. U. S. A. 1996, 93, 2493-2498.

(41) Shapovalov, M. V.; Dunbrack, R. L. A Smoothed BackboneDependent Rotamer Library for Proteins Derived from Adaptive Kernel Density Estimates and Regressions. Structure 2011, 19, 844858.

(42) Chen, V. B.; Arendall, W. B., 3rd; Headd, J. J.; Keedy, D. A.; Immormino, R. M.; Kapral, G. J.; Murray, L. W.; Richardson, J. S.; Richardson, D. C. MolProbity: All-atom Structure Validation for Macromolecular Crystallography. Acta Crystallogr., Sect. D: Biol. Crystallogr. 2010, 66, 12-21.

(43) Sondergaard, C. R.; Olsson, M. H.; Rostkowski, M.; Jensen, J. H. Improved Treatment of Ligands and Coupling Effects in Empirical 
Calculation and Rationalization of $\mathrm{p} K_{\mathrm{a}}$ Values. J. Chem. Theory Comput. 2011, 7, 2284-2295.

(44) Ryckaert, J.-P.; Ciccotti, G.; Berendsen, H. J. C. Numerical Integration of the Cartesian Equations of Motion of a System with Constraints: Molecular Dynamics of n-Alkanes. J. Comput. Phys. 1977, 23, 327-341.

(45) Darden, T.; York, D.; Pedersen, L. Particle Mesh Ewald: An N $\log (\mathrm{N})$ Method for Ewald Sums in Large Systems. J. Chem. Phys. 1993, 98, 10089-10092.

(46) Roe, D. R.; Cheatham, T. E. 3rd, PTRAJ and CPPTRAJ: Software for Processing and Analysis of Molecular Dynamics Trajectory Data. J. Chem. Theory Comput. 2013, 9, 3084-3095.

(47) R Core Team: A language and environment for statistical computing. R Foundation for Statistical Computing; https://www.Rproject.org/.

(48) Pedersen, A. K.; Peters, G. H.; Møller, K. B.; Iversen, L. F.; Kastrup, J. S. Water-Molecule Network and Active-Site Flexibility of Apo Protein Tyrosine Phosphatase 1B. Acta Crystallogr., Sect. D: Biol. Crystallogr. 2004, 60, 1527-1534.

(49) Cui, D. S.; Lipchock, J. M.; Brookner, D.; Loria, J. P. Uncovering the Molecular Interactions in the Catalytic Loop That Modulate the Conformational Dynamics in Protein Tyrosine Phosphatase 1B. J. Am. Chem. Soc. 2019, 141, 12634-12647.

(50) Whitten, S. T.; Garcia-Moreno, E. B.; Hilser, V. J. Local Conformational Fluctuations Can Modulate the Coupling Between Proton Binding and Global Structural Transitions in Proteins. Proc. Natl. Acad. Sci. U. S. A. 2005, 102, 4282-4287.

(51) Knapp, B.; Ospina, L.; Deane, C. M. Avoiding False Positive Conclusions in Molecular Simulation: The Importance of Replicas. J. Chem. Theory Comput. 2018, 14, 6127-6138.

(52) Liao, Q.; Kulkarni, Y.; Sengupta, U.; Petrović, D.; Mulholland, A. J.; van der Kamp, M. W.; Strodel, B.; Kamerlin, S. C. L. Loop Motion in Triosephosphate Isomerase Is Not a Simple Open and Shut Case. J. Am. Chem. Soc. 2018, 140, 15889-15903.

(53) Maria-Solano, M. A.; Iglesias-Fernández, J.; Osuna, S. Deciphering the Allosterically Driven Conformational Ensemble in Tryptophan Synthase Evolution. J. Am. Chem. Soc. 2019, 141, 1304913056.

(54) Smith, Z.; Ravindra, P.; Wang, Y.; Cooley, R.; Tiwary, P. Discovering Protein Conformational Flexibility through ArtificialIntelligence-Aided Molecular Dynamics. J. Phys. Chem. B 2020, 124, $8221-8229$.

(55) Hjortness, M. K.; Riccardi, L.; Hongdusit, A.; Zwart, P. H.; Sankaran, B.; De Vivo, M.; Fox, J. M. Evolutionarily Conserved Allosteric Communication in Protein Tyrosine Phosphatases. Biochemistry 2018, 57, 6443-6451.

(56) Ahuja, L. G.; Taylor, S. S.; Kornev, A. P. Tuning the "Violin" of Protein Kinases: The Role of Dynamics-Based Allostery. IUBMB Life 2019, 71, 685-696.

(57) Taylor, S. S.; Kornev, A. P. Protein Kinases: Evolution of Dynamic Regulatory Proteins. Trends Biochem. Sci. 2011, 36, 65-77. 